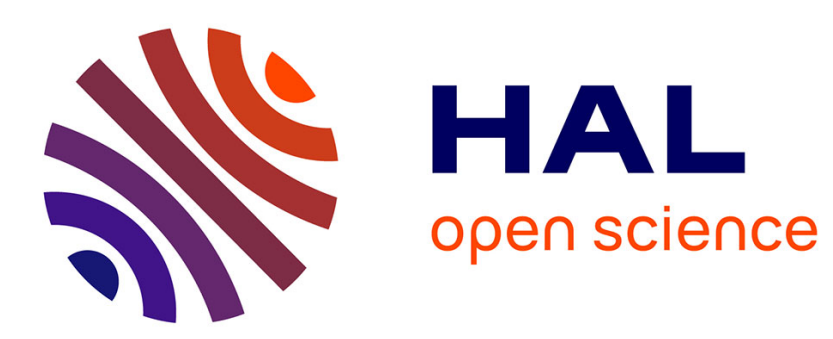

\title{
Diagnosis and prediction of vibration from railway trains
}

Benoit Picoux, Donatien Le Houedec

\section{To cite this version:}

Benoit Picoux, Donatien Le Houedec. Diagnosis and prediction of vibration from railway trains. Soil Dynamics and Earthquake Engineering, 2005, 25 (12), pp.905-921. 10.1016/j.soildyn.2005.07.002 . hal-01006743

\section{HAL Id: hal-01006743 \\ https://hal.science/hal-01006743}

Submitted on 16 Jun 2014

HAL is a multi-disciplinary open access archive for the deposit and dissemination of scientific research documents, whether they are published or not. The documents may come from teaching and research institutions in France or abroad, or from public or private research centers.
L'archive ouverte pluridisciplinaire HAL, est destinée au dépôt et à la diffusion de documents scientifiques de niveau recherche, publiés ou non, émanant des établissements d'enseignement et de recherche français ou étrangers, des laboratoires publics ou privés. 


\title{
Diagnosis and prediction of vibration from railway trains
}

\author{
B. Picoux ${ }^{\mathrm{a}}$, D. Le Houédec ${ }^{\mathrm{b}, 1}$ \\ a 3MSGC Laboratory, Civil Engineering Department, Bd J. Derche, 19300 Egletons, France \\ ${ }^{\mathrm{b}}$ GeM Laboratory, UMR CNRS 6183, Ecole Centrale of Nantes, 1 rue de la Noë, 44321 Nantes Cedex 3, France
}

In the North West of France, more particularly in the region of the Somme Bay, where the ground is constituted mainly of peat, observation of the surface of the soil near railway tracks has revealed high levels of displacement. This paper, contains a prediction model and diagnosis of vibration near the track. A model of a railway track on layered ground subjected to a moving train has been built and the calculation method uses Fourier transform formalism for a semi-analytical solution in the wave number domain. It includes all elements of the track and allows a parametric analysis of its different elements and evaluation of vertical displacement according to the speed, weight and composition of each train. The diagnosis has been performed with in situ measurements and with the aim of the validation of the model. A parameter study of the ground undertaken by seismic measurements shows a critical speed close to $100 \mathrm{~m} / \mathrm{s}$ while the studied trains are moving with subRayleigh speeds. Measurements give us a lot of information about lateral and vertical acceleration on the soil's surface and parts of the track. For high speeds and freight trains, displacement reaches more than $10 \mathrm{~mm}$.

Keywords: Wave propagation; Railway track; Moving loads (train); Soft soils; In situ measurements; Diagnosis

\section{Introduction}

\subsection{Background to the problem}

The consequences of railway traffic, in terms of environmental damage (vibration, noise and various polluters) are all the more important in view of the fact that the speed of trains has increased. This increase in speed is particularly sought after for railway trains like the high speed T.G.V. in France. Concerning vibration, the effect of this speed is directly connected to its relative position from waves propagating through the ground (especially superficial Rayleigh waves). For relatively soft ground (clay, peat, ...), a Rayleigh wave speed of less than $100 \mathrm{~m} / \mathrm{s}$ is possible, and in this case the super-Rayleigh regime is reached for trains moving at high speed. This situation induces high levels of displacement in the rail and at the ground's surface, which has no impact on the comfort of passengers, but is worrying for the wear and tear of the tracks and neighbouring structures.

\subsection{General outline}

The French project 'PREDIT' includes the design of simulation and diagnosis tools which enable us to simulate and characterize all phenomena concerned with ground traffic as precisely as possible. In fact, before the development of numerical methods, available means were limited to relatively simple models (for example, a beam on an elastic foundation subjected to a moving load) and generally taking into account only a part of the whole coupled problem: train-track-ground. Despite some simplified hypotheses, the objective of this study is, therefore, to ensure better interaction thanks to a more accurate model and including, both specific experiments (the French national railway company SNCF for the track, SoletancheBachy for the ground) and published results.

As well as this model, we must refer to an experimental in situ validation. Recordings have been made to build a usable experimental data bank for the comparison between in situ measurements and numerical results of 
the simulation. For this comparison, it is necessary to obtain all the data to test the model. Therefore, it was necessary to first choose suitable data to perform numerical simulations. Ground parameters are deduced from seismic recordings and borings. Different models have been tried: semi-infinite half space and one or two soft layers on substratum.

\subsection{Review of published results}

Many theoretical 2D models have been studied for semiinfinite half space. Alabi (cf [1] and [2]) has studied the effects of a line load on the surface of the ground and shown the high levels of vertical displacement on the surface. In order to take layered ground into account, De Barros and Luco (cf [3] and [4]) have developed a semi-analytical model for a point load moving on a viscoelastic multilayered half-space (details in [5]), using the determination and combination of stiffness matrices obtained for each layer. This model is based on a double Fourier transform and shows the presence of Mach cones in the first layer for super-Rayleigh speeds. This model was then used by Gunaratine and Sanders for the road [6] and by Auersh for track excitation [7]. It is also used by Jones and Petyt for rectangular loads or strip loads with different ground conditions: half space [8], elastic layers on a half space [9] or on a rigid foundation [10].

A track is composed of two rails lying on sleepers (wood or concrete) joined by railpads. The rail provides the contact between the train wheel and the track. Vibrations from trains propagating from the rail to the ballast going through the sleepers, strains these same elements. This distribution was mainly studied by Krylov, cf [11]. In fact the rail represents the main source of excitation. For a railway system, the excitation on the ground's surface is generated mainly by irregular contact between the wheel and the rail due to manufacturing defects or wear and tear. With a high number of trains, the properties of the track amplify the excitation. The difficulty of the model comes from the fact that the track has to be built with materials described by complex constitutive Equation (like ballast) (e.g. by Gudehus [12]).

Boundary element models (used by Lombaert et al. [13]), finite element models (used by Hall [14] for example) can be also used. The rail is then modelled using beam elements (Rayleigh-Timoshenko beam) supported by rigid sleepers. Each sleeper has two degrees of freedom: rotation and translation and lies on the ballast. The sleepers interact with each other by damping-shock absorbers (representing the railpads). The excitation is modelled as a series of point loads representing the train moving at constant speed. The load acts on a node along the beam with a time condition corresponding to the speed of the train and the distance between each node. The problem is then formulated in the wave number domain. This model thus enables us to take the non-linearities of the ground and the ballast into account. Finally, by modal analysis or analysis of the dispersion, parametric amplification notion is justified.
Coupled finite-boundary element models (Andersen et al. [15]) can also be used. In this case, finite elements are used to model the structure of the track and the ground is described using a boundary element model. The coupling of the finite element and the boundary element zones is carried out in a context of nodal forces, i.e. in a finite element manner. The coupled model is applied to the study of the screening effect due to open or in-filled trenches along the track. To reduce ground vibration caused by rail traffic, Massarch [16] has also studied the isolation effect of open or gas-filled trenches based on field measurements.

For Sheng et al. [17] the continuously welded rail is modelled as an Euler beam lying on a Timoshenko beam representing the sleepers. The ballast is modelled by vertical stiffness and a uniformally distributed mass. This model takes two loading modes into account (static and dynamic) and shows the importance of the dynamic regime in the case of super-Rayleigh for the amplification of vibration [18]. For low frequencies, the train-track interaction as well as the inertia of the rail and sleepers are not considered. The soil model frequently used for simulation (for example, in [19]) is similar to that developed by Jones and Petyt [20] and the track model by Jones et al. [21]. The moving vertical excitation includes a constant component and a harmonic one simulating irregularities in the track (single frequency). Forces due to track-soil interaction are roughly estimated, as in [22], and become significant for frequencies less than $40 \mathrm{~Hz}$. Dinkel et al. [23] calculated the linear response of the ground using methods which sometimes include wavelet algorithms.

The analytical solution of the problem has been obtained by Felszeghy in [24] using a simplified rail-ballast-ground model using a beam (Euler-Bernoulli or Timoshenko) supported by an elastic foundation (springs and dampers). An equivalent 3D numerical model has been also compared to high speed train measurements in Ledsgard (Sweden) by Paolucci et al. [25]. Dieterman and Metrikine [26] have shown that two critical speeds exist in the case of a beam on homogeneous ground, excited by a harmonic load, one equal to the speed of the Rayleigh wave in the ground and one other close to the speed of the Rayleigh wave, controlled by the stiffness and the mass of the beam. Moreover, energy is transferred from the beam to the ground when the phase speed in the track is less than the Rayleigh speed of the ground. Krylov [27] has deduced that the displacement amplitude on the ground's surface depends on the period and the number of sleepers (also found by Heckl et al. in [28]). In the case of multilayered ground, waves are scattered with a higher penetration of energy into the ground.

Finally, the heterogeneous effect of the ballast can be considered. This was done by Suiker et al. ([29] and [30]) for example. The ballast is then no longer modelled as a classic elastic continuous medium but to a Cosserat model including rotations-translation and inertia of the granular material (depending on the size of the grains). 


\section{Mathematical model}

Two dimensional models are not able to reproduce mechanisms of wave propagation in the ground correctly since the loading zone represents a reduced area compared to infinite ground surface, thus excluding the hypothesis of plane deformation. This limitation has motivated researchers to obtain solutions using 3D models (see Fig. 1).

\subsection{Description of the model}

The calculation method uses the Fourier transform formalism for a semi-analytical solution in the wave number domain. The stiffness matrix for layered ground was written and accompanied by a fitted phase argument in Helmoltz functions. This provides us a fast numerical approach to the problem. Equations are written in the wave number domain as well as for steady state solutions. An inverse numerical Fourier transform is then applied when the matrix equation of the whole system (train-track-soil) has been solved.

\subsection{Analytical solution to the $3 D$ half-space problem}

Soil is homogeneous and isotropic. Intrinsic parameters for a layer of ground are Young's modulus E, Poisson's ratio $\nu$, density $\rho$, hysteretic damping factor $\eta$ and thickness $h$. For layered ground, a rigidity matrix connecting stress to displacement at the interface of each layer can be obtained.

For a displacement vector $\vec{U}$ which has components $(\mathbf{u}, \mathbf{v}$, w), the behaviour of the half-space is described by Navier's elastodynamic equations. In the absence of body force, one obtains the following vector equation:

$(\lambda+\mu) \nabla(\nabla \vec{U})+\mu \nabla^{2} \vec{U}=\rho \frac{\partial^{2} \vec{U}}{\partial t^{2}}$

where $\lambda$ et $\mu$ are Lamé's constants, $\nabla$ is the gradient operator and $\nabla^{2}$ is the laplacian operator.
The stress-strain relation can be expressed as:

$$
\tau_{i j}=\lambda \delta_{i j} \Delta \vec{U}+\mu\left(\frac{\partial \mathbf{u}_{i}}{\partial \mathbf{x}_{j}}+\frac{\partial \mathbf{u}_{j}}{\partial \mathbf{x}_{i}}\right)
$$

where $\delta_{\mathrm{ij}}$ is the Kronecker delta, $\mathbf{u}_{i}$ and $\mathbf{x}_{i}$ are the $i$ th component of the vectors $(\mathbf{u}, \mathbf{v}, \mathbf{w})$ and $\left(\mathbf{x}_{1}, \mathbf{x}_{2}, \mathbf{x}_{3}\right)$ and $\Delta$ is the dilatation defined by $\left(\left(/\left(\mathbf{x}_{1}+\left(/\left(\mathbf{x}_{2}+\left(/ / \mathbf{x}_{3}\right)\right.\right.\right.\right.\right.$.

In the $3 \mathrm{D}$ model, the equation of motion for the soil is satisfied by writing [31]:

$$
\begin{aligned}
& \mathbf{u}=\frac{\partial \phi}{\partial x_{1}}+\frac{\partial^{2} \psi}{\partial x_{1} \partial x_{3}} ; \quad v=\frac{\partial \phi}{\partial x_{2}}+\frac{\partial^{2} \psi}{\partial x_{2} \partial x_{3}} ; \\
& w=\frac{\partial \phi}{\partial x_{3}}+\frac{\partial^{2} \psi}{\partial x_{3}^{2}}-\frac{1}{c_{S}} \frac{\partial^{2} \psi}{\partial t^{2}} .
\end{aligned}
$$

where $\phi$ comes from compression waves (particle movement in the direction of wave propagation) and $\psi$ of shear waves (particle movement perpendicular to the direction of propagation) as in these equations:

$$
\nabla^{2} \phi=\frac{1}{c_{\mathrm{p}}^{2}} \frac{\partial^{2} \phi}{\partial t^{2}} \text { and } \nabla^{2} \psi=\frac{1}{c_{\mathrm{s}}^{2}} \frac{\partial^{2} \psi}{\partial t^{2}}
$$

where $c_{\mathrm{p}}$ and $c_{\mathrm{s}}$ are compression and shear wave speed, respectively.

Then, Eq. (2) and boundary conditions allow us to write the following equations in $\left(0, \mathbf{x}_{1}, \mathbf{x}_{2}, \mathbf{x}_{3}\right)$ relative to the soil:

$$
\begin{aligned}
\tau_{33}\left(\mathbf{x}_{1}, \mathbf{x}_{2}, \mathbf{x}_{3}, t\right) & =\frac{\lambda}{c_{\mathrm{P}}^{2}} \frac{\partial^{2} \phi}{\partial t^{2}}+2 \mu\left(\frac{\partial^{2} \phi}{\partial \mathbf{x}_{3}^{2}}+\frac{\partial^{3} \psi}{\partial \mathbf{x}_{3}^{3}}\right)-2 \rho \frac{\partial^{3} \psi}{\partial \mathbf{x}_{3} \partial t^{2}} \\
& = \begin{cases}-\frac{F_{\mathrm{B}}\left(x_{1}, t\right)}{2 L_{\mathrm{BAL}}} & \text { if }\left|x_{2}\right| \leq 2 L_{\mathrm{BAL}} \\
0 & \text { else }\end{cases}
\end{aligned}
$$

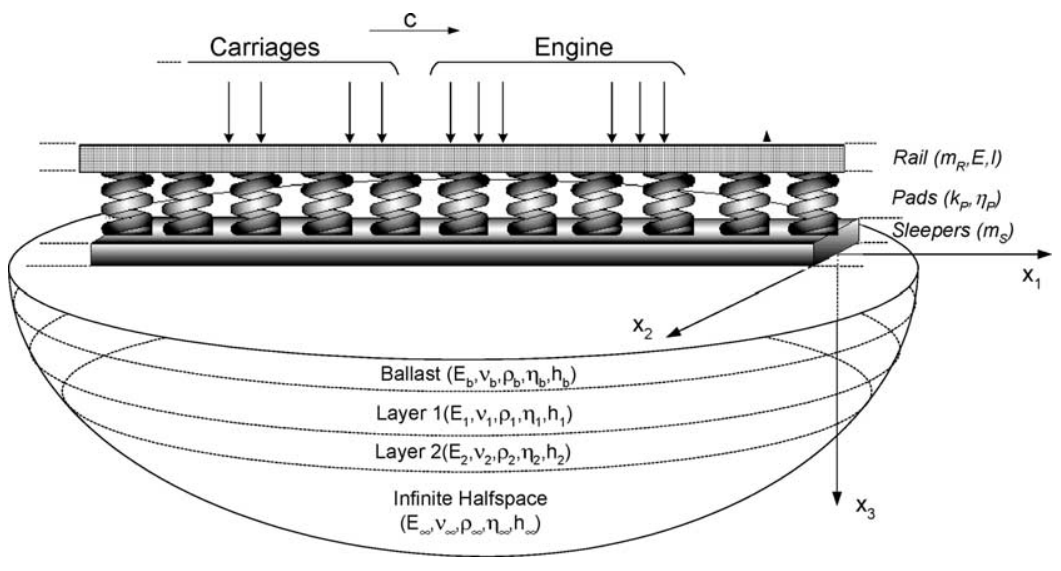

Fig. 1. Representation of the 3D model. 
$\tau_{13}\left(\mathbf{x}_{1}, \mathbf{x}_{2}, \mathbf{x}_{3}, t\right)=2 \mu\left(\frac{\partial^{2} \phi}{\partial \mathbf{x}_{1} \partial \mathbf{x}_{3}}+\frac{\partial^{3} \psi}{\partial \mathbf{x}_{1} \partial \mathbf{x}_{3}^{2}}\right)-\rho \frac{\partial^{3} \psi}{\partial \mathbf{x}_{1} \partial t^{2}}=0$

$\tau_{23}\left(\mathbf{x}_{1}, \mathbf{x}_{2}, \mathbf{x}_{3}, t\right)=2 \mu\left(\frac{\partial^{2} \phi}{\partial \mathbf{x}_{2} \partial \mathbf{x}_{3}}+\frac{\partial^{3} \psi}{\partial \mathbf{x}_{2} \partial \mathbf{x}_{3}^{2}}\right)-\rho \frac{\partial^{3} \psi}{\partial \mathbf{x}_{2} \partial t^{2}}=0$

where $F_{\mathrm{B}}\left(\mathbf{x}_{1}, t\right)$ is the vertical force acting on the ballast, $L_{\mathrm{BAL}}$ is the ballast width.

Changes of functions and variables are performed:

$$
\left\{\begin{array}{l}
x=x_{1}-c t ; y=x_{2} ; z=x_{3} \\
w(\beta, t)=w^{*}(\beta) \mathrm{e}^{i \Omega_{0} t} ; F(\beta, t)=F^{*}(\beta) \mathrm{e}^{i \Omega_{0} t}
\end{array}\right.
$$

where $c$ is the speed of the train, $\Omega_{0}$ is the excitation frequency, * indicates that we study the steady state solution and $\beta$ and $\gamma$ are the wave numbers relative to $x$ and $y$.

A double Fourier transform $(x, y) \rightarrow(\beta, \gamma)$ gives:
Stress continuity for $z=0$ is given by:

$$
\tau_{z z}(x, y, 0, t)=-\frac{F_{\mathrm{B}}(x, t)}{2_{\mathrm{LBAL}}}
$$

So, displacement below the ballast is written as follows:

$$
\begin{aligned}
\bar{w}_{\mathrm{B}}^{*}(\beta)= & -\frac{\bar{F}_{\mathrm{B}}^{*}(\beta)}{\pi \mu} \int_{0}^{\infty} \frac{\left(\Omega_{0}-\beta c\right)^{2}}{c_{\mathrm{S}}^{2}} \\
& \times \frac{\alpha_{\mathrm{P}}}{\left(\alpha_{\mathrm{S}}^{2}+\beta^{2}+\gamma^{2}\right)^{2}-4 \alpha_{\mathrm{P}} \alpha_{\mathrm{S}}\left(\beta^{2}+\gamma^{2}\right)} \\
& \times \frac{\sin \gamma L_{\mathrm{BAL}}}{\gamma L_{\mathrm{BAL}}} \mathrm{d} \gamma
\end{aligned}
$$

The equation for a rail represented by an Euler beam is written:

$$
\left\{\begin{array}{l}
\frac{\lambda}{c_{\mathrm{P}}^{2}}\left(\Omega_{0}-\beta c\right)^{2} \bar{\phi} *(\beta, \gamma)+2 \mu\left(\frac{\partial^{2} \bar{\phi} *(\beta, \gamma)}{\partial z^{2}}+\frac{\partial^{3} \bar{\psi} *(\beta, \gamma)}{\partial z^{3}}\right)-2 \rho\left(\Omega_{0}-\beta c\right)^{2} \frac{\partial \bar{\psi} *(\beta, \gamma)}{\partial z}=-\bar{F}_{B}^{*}(\beta) \frac{\sin \gamma L_{\mathrm{BAL}}}{\gamma L_{\mathrm{BAL}}} \\
2 \mu \frac{\partial \bar{\phi} *(\beta, \gamma)}{\partial z}+2 \mu \frac{\partial^{2} \bar{\psi} *(\beta, \gamma)}{\partial z^{2}}+\rho\left(\Omega_{0}-\beta c\right)^{2} \bar{\psi} *(\beta, \gamma)=0
\end{array}\right.
$$

In these equations " ${ }^{\text {"- }}$ refers to function in the wave number domain.

Solutions with no propagating waves in negative $z$ direction (no reflection) for this last set of equation are:

$\bar{\phi}^{*}(\beta, \gamma)=A(\beta, \gamma) \mathrm{e}^{-\alpha_{\mathrm{p}} z}$ and $\bar{\psi}^{*}(\beta, \gamma)=B(\beta, \gamma) \mathrm{e}^{-\alpha_{\mathrm{S}} z}$

$\begin{aligned} & \text { where } \\ & \left(\frac{\Omega_{0}-\beta c}{c_{\mathrm{S}}}\right)^{2}\end{aligned} \alpha_{\mathrm{P}}^{2}=\beta^{2}+\gamma^{2}-\left(\frac{\Omega_{0}-\beta c}{c_{\mathrm{P}}}\right)^{2} \quad$ and $\quad \alpha_{\mathrm{S}}^{2}=\beta^{2}+\gamma^{2}-$ Displacement on the soil's surface is given by:

$\bar{w}^{*}=-\alpha_{\mathrm{P}} \bar{A}(\beta, \gamma)+\alpha_{\mathrm{S}}^{2} \bar{B}(\beta, \gamma)+\left(\frac{\Omega_{0}-\beta c}{c_{\mathrm{S}}}\right)^{2} \bar{B}(\beta, \gamma)$

By solving previous equation, vertical displacement in the wave number domain and on the soil's surface $(z=0)$ is written as follows:

$$
\begin{aligned}
\bar{w}^{*}= & -\frac{\bar{F}_{\mathrm{B}}^{*}(\beta)}{\mu} \frac{\left(\Omega_{0}-\beta c\right)^{2}}{c_{\mathrm{S}}^{2}} \\
& \times \frac{\alpha_{\mathrm{P}}}{\left(\alpha_{\mathrm{S}}^{2}+\beta^{2}+\gamma^{2}\right)^{2}-4 \alpha_{\mathrm{P}} \alpha_{\mathrm{S}}\left(\beta^{2}+\gamma^{2}\right)} \frac{\sin \gamma L_{\mathrm{BAL}}}{\gamma L_{\mathrm{BAL}}}
\end{aligned}
$$

The aim is to determine $\bar{w}^{*}$ on the soil's surface and to deduce $w$ with the help of inverse numerical Fourier transform. Thus, it is necessary to calculate $\bar{F}_{\mathrm{B}}^{*}(\beta)$.

$$
\begin{aligned}
& \mathrm{EI} \frac{\partial^{4} w_{\mathrm{R}}\left(x_{1}, t\right)}{\partial x_{1}^{4}}+m_{\mathrm{R}} \frac{\partial^{2} w_{\mathrm{R}}\left(x_{1}, t\right)}{\partial t^{2}}+k_{\mathrm{P}}\left[w_{\mathrm{R}}\left(x_{1}, t\right)-w_{\mathrm{S}}\left(x_{1}, t\right)\right] \\
& \quad= \begin{cases}\frac{P_{0} \mathrm{e}^{i Q_{0} t}}{2 b} & \text { pour }\left|x_{1}-c t\right|<b \\
0 & \text { sinon }\end{cases}
\end{aligned}
$$

where $x_{1}-c t$ indicates the load displacement.

Sleepers are represented by a continuous mass in the equation:

$m_{\mathrm{S}} \frac{\partial^{2} w_{\mathrm{S}}\left(x_{1}, t\right)}{\partial t^{2}}+k_{\mathrm{P}}\left[w_{\mathrm{S}}\left(x_{1}, t\right)-w_{\mathrm{R}}\left(x_{1}, t\right)\right]=-F_{\mathrm{S}}\left(x_{1}, t\right)$

where $F_{\mathrm{S}}$ is the rail force on the sleepers.

At the top and bottom of the ballast, the system is written as:

$$
\begin{aligned}
& \frac{m_{\mathrm{B}}}{6}\left[2 \frac{\partial^{2} w_{\mathrm{S}}\left(x_{1}, t\right)}{\partial t^{2}}+\frac{\partial^{2} w_{\mathrm{B}}\left(x_{1}, t\right)}{\partial t^{2}}\right]+k_{\mathrm{B}}\left[w_{\mathrm{S}}\left(x_{1}, t\right)-w_{\mathrm{B}}\left(x_{1}, t\right)\right] \\
& \quad=F_{\mathrm{S}}\left(x_{1}, t\right)
\end{aligned}
$$




$$
\begin{aligned}
& \frac{m_{\mathrm{B}}}{6}\left[\frac{\partial^{2} w_{\mathrm{S}}\left(x_{1}, t\right)}{\partial t^{2}}+2 \frac{\partial^{2} w_{\mathrm{B}}\left(x_{1}, t\right)}{\partial t^{2}}\right]+k_{\mathrm{B}}\left[-w_{\mathrm{S}}\left(x_{1}, t\right)\right. \\
& \left.\quad+w_{\mathrm{B}}\left(x_{1}, t\right)\right]=-F_{\mathrm{B}}\left(x_{1}, t\right)
\end{aligned}
$$

where $F_{\mathrm{B}}$ is the ballast force on the soil.

Damping $\eta_{\mathrm{i}}$ in pads and ballast is taken into account [32] in stiffness as

$k_{i}^{*}=k_{i}\left[1+i \eta_{i} \operatorname{sign}\left(\Omega_{0}-\beta c\right)\right]$.

Using Eq. (7), the following set of equations relative to the track is obtained:

$$
\left\{\begin{array}{l}
A_{1}(\beta) \bar{w}_{\mathrm{R}}^{*}(\beta)-k_{\mathrm{P}} \bar{w}_{\mathrm{S}}^{*}(\beta)=A_{2}(\beta) \\
-k_{\mathrm{p}} \bar{w}_{\mathrm{R}}^{*}(\beta)+A_{3}(\beta) \bar{w}_{\mathrm{S}}^{*}(\beta)+A_{4}(\beta) \bar{w}_{\mathrm{B}}^{*}(\beta)=0 \\
A_{4}(\beta) \bar{w}_{\mathrm{S}}^{*}(\beta)+A_{5}(\beta) \bar{w}_{\mathrm{B}}^{*}(\beta)=-\bar{F}_{\mathrm{B}}^{*}(\beta) \\
\bar{w}_{\mathrm{B}}^{*}(\beta)=A_{6}(\beta) \bar{F}_{\mathrm{B}}^{*}(\beta)
\end{array}\right.
$$

where

$$
\begin{aligned}
A_{1}(\beta)= & \mathrm{EI} \beta^{4}-(\Omega-\beta c)^{2} m_{\mathrm{R}}+k_{\mathrm{P}} ; \quad A_{2}(\beta)=P_{0} ; \\
A_{4}(\beta)= & -(\Omega-\beta c)^{2} \frac{m_{\mathrm{B}}}{6}-k_{\mathrm{B}} ; \\
A_{3}(\beta)= & -m_{\mathrm{S}}(\Omega-\beta c)^{2}+k_{\mathrm{P}}+k_{\mathrm{B}}-\frac{m_{\mathrm{B}}}{3}(\Omega-\beta c)^{2} ; \\
A_{5}(\beta)= & -(\Omega-\beta c)^{2} \frac{m_{\mathrm{B}}}{3}+k_{\mathrm{B}} ; \\
A_{6}(\beta)= & -\frac{1}{\pi \mu} \int_{0}^{\infty} \frac{\left(\Omega_{0}-\beta c\right)^{2}}{c_{\mathrm{S}}^{2}} \\
& \times \frac{\alpha_{\mathrm{P}}}{\left(\alpha_{\mathrm{S}}^{2}+\beta^{2}+\gamma^{2}\right)^{2}-4 \alpha_{\mathrm{P}} \alpha_{\mathrm{S}}\left(\beta^{2}+\gamma^{2}\right)} \\
& \times \frac{\sin \gamma L_{\mathrm{BAL}}}{\gamma L_{\mathrm{BAL}}} \mathrm{d}
\end{aligned}
$$

Solving the Eq. (18) leads to the force expression:

$\bar{F}_{\mathrm{B}}^{*}(\beta)=\frac{A_{2}(\beta) A_{4}(\beta) k_{\mathrm{P}}}{A_{1}(\beta) A_{6}(\beta) A_{4}^{2}(\beta)\left(A_{3}(\beta) A_{1}(\beta)-k_{\mathrm{P}}^{2}\right)\left(1+A_{5}(\beta) A_{6}(\beta)\right)}$

and transformed displacement is finally given by:

$$
\begin{aligned}
\bar{w}^{*}= & -\frac{\bar{F}_{\mathrm{B}}^{*}(\beta)}{\mu} \frac{\left(\Omega_{0}-\beta c\right)^{2}}{c_{\mathrm{S}}^{2}} \\
& \times \frac{\alpha_{\mathrm{P}}}{\left(\alpha_{\mathrm{S}}^{2}+\beta^{2}+\gamma^{2}\right)^{2}-4 \alpha_{\mathrm{P}} \alpha_{\mathrm{S}}\left(\beta^{2}+\gamma^{2}\right)} \\
& \times \frac{\sin \gamma L_{\mathrm{BAL}}}{\gamma L_{\mathrm{BAL}}} .
\end{aligned}
$$

\subsection{Semi-analytical solution to the $3 D$ multilayered problem}

In this part, the previous model (Fig. 1) is used with several ground layers below the ballast. A relation between displacements and stresses can be written into a matrix formulation.

First the dilatation $\theta$ is introduced as:

$\bar{\theta}^{*}=i \beta \bar{u}^{*}+i \gamma \bar{v}^{*}+\frac{\mathrm{d} \bar{w}^{*}}{\mathrm{~d} z}=\bar{A} \mathrm{e}^{\alpha_{\mathrm{P}}(z-h)}+\bar{B} \mathrm{e}^{-\alpha_{\mathrm{P}} z}$

Wave equation appear using $\bar{\theta}^{*}$ :

$$
\left(\frac{\mathrm{d}^{2}}{\mathrm{~d} z^{2}}-\alpha_{\mathrm{S}}^{2}\right)\left\{\begin{array}{l}
\bar{u}^{*} \\
\bar{v}^{*} \\
\bar{w}^{*}
\end{array}\right\}=-\frac{\lambda+\mu}{\mu}\left\{\begin{array}{c}
i \beta\left(\bar{A} \mathrm{e}^{\alpha_{\mathrm{P}}(z-h)}+\bar{B} \mathrm{e}^{-\alpha_{\mathrm{P}} z}\right) \\
i \gamma\left(\bar{A} \mathrm{e}^{\alpha_{\mathrm{P}}(z-h)}+\bar{B} \mathrm{e}^{-\alpha_{\mathrm{P}} z}\right) \\
\alpha_{\mathrm{P}}\left(\bar{A} \mathrm{e}^{\alpha_{\mathrm{P}}(z-h)}-\bar{B} \mathrm{e}^{-\alpha_{\mathrm{P}} z}\right)
\end{array}\right\}
$$

where $\alpha_{\mathrm{P}}$ and $\alpha_{\mathrm{S}}$ are defined in (9).

Solutions to this last equation are expressed as:

$$
\begin{aligned}
\left.\begin{array}{c}
\bar{u}^{*} \\
\bar{v}^{*} \\
\bar{w}^{*}
\end{array}\right\}= & -\frac{\bar{A} \mathrm{e}^{\alpha_{\mathrm{P}}(z-h)}}{\left(\frac{\Omega_{0}-\beta c}{c_{\mathrm{P}}^{2}}\right)^{2}}\left\{\begin{array}{c}
-i \beta \\
-i \gamma \\
-\alpha_{\mathrm{P}}
\end{array}\right\}+\frac{\bar{B} \mathrm{e}^{\alpha_{\mathrm{P}}(z-h)}}{\left(\frac{\Omega_{0}-\beta c}{c_{\mathrm{P}}^{2}}\right)^{2}}\left\{\begin{array}{c}
-i \beta \\
-i \gamma \\
\alpha_{\mathrm{P}}
\end{array}\right\} \\
& +\mathrm{e}^{\alpha_{\mathrm{S}}(z-h)}\left\{\begin{array}{l}
\bar{C} \\
\bar{D} \\
-\frac{i}{\alpha_{\mathrm{S}}}(\beta \bar{C}+\gamma \bar{D})
\end{array}\right\} \\
& +\mathrm{e}^{-\alpha_{\mathrm{S}} z}\left\{\begin{array}{l}
\bar{E} \\
\bar{F} \\
\frac{i}{\alpha_{\mathrm{S}}}(\beta \bar{E}+\gamma \bar{F})
\end{array}\right\}
\end{aligned}
$$

For a layer of thickness $h$, displacement is written in terms of the constants $\bar{A}, \bar{B}, \bar{C}, \bar{D}, \bar{E}$ and $\bar{F}$ :

$\left\{\bar{U}^{*}\right\}=\left\{\bar{u}_{0}^{*} \bar{v}_{0}^{*} i w_{0}^{*} \bar{u}_{\mathrm{h}}^{*} \bar{v}_{\mathrm{h}}^{*} i \bar{w}_{\mathrm{h}}^{*}\right\}=[Q]\{\bar{A} \bar{B} \bar{C} \bar{D} \bar{E} \bar{F}\}^{T}$

Longitudinal, lateral and vertical stresses are given, respectively, by:

$$
\begin{aligned}
& \frac{\bar{\tau}_{x z}^{*}}{\mu}=\frac{\mathrm{d} \bar{u}^{*}}{\mathrm{~d} z}+i \beta \bar{w}^{*} ; \quad \frac{\bar{\tau}_{y z}^{*}}{\mu}=\frac{\mathrm{d} \bar{v}^{*}}{\mathrm{~d} z}+i \gamma \bar{w}^{*} ; \\
& \frac{\bar{\tau}_{z z}^{*}}{\mu}=\frac{\lambda}{\mu} \bar{\theta}^{*}+2 \frac{\mathrm{d} \bar{w}^{*}}{\mathrm{~d} z}
\end{aligned}
$$


Similarly, for the same layer, stress can be expressed in terms of the previous constants:

$$
\begin{aligned}
\left\{\bar{\Sigma}^{*}\right\} & =\left\{-\bar{\tau}_{x z, 0}^{*}-\bar{\tau}_{y z, 0}^{*}-i \bar{\tau}_{z z, 0}^{*} \bar{\tau}_{x z, h}^{*} \bar{\tau}_{y z, h}^{*} i \bar{\tau}_{z z, h}^{*}\right\} \\
& =[S]\{\bar{A} \bar{B} \bar{C} \bar{D} \bar{E} \bar{F}\}^{T}
\end{aligned}
$$

For the ground, the 3D stiffness matrix is the combination of the stiffness matrices of each layer:

$$
\left[\begin{array}{l}
\text { Stiffness } \\
\text { Matrix }
\end{array}\right]\{\bar{U} *\}=\{\bar{\Sigma} *\}
$$

where $\{\bar{U} *\}$ is the transformed displacement vector and $\{\bar{\Sigma} *\}$ is the stress vector.

Consequently, the displacement vector $\{\bar{U} *\}$ is given by:

$\{\bar{U} *\}=\left[\begin{array}{l}\text { Stiffness } \\ \text { Matrix }\end{array}\right]^{-1}\{\bar{\Sigma} *\}=[B]\{\bar{\Sigma} *\}$

where $[B]$ is the inverse stiffness matrix for multilayered ground.

The general stiffness matrix is obtained from matrices $[Q],[S]$ and $[R]$ detailed in the Appendix.

As no lateral and longitudinal stress is considered in the present problem: $\bar{\tau}_{x z}=\bar{\tau}_{y z}=0$, displacement is obtained as follows:

$$
\begin{aligned}
& \bar{u} *(0)=-i B_{31} \bar{\tau}_{z z}^{*}(0) ; \quad \bar{v} *(0)=-i B_{32} \bar{\tau}_{z z}^{*}(0) ; \\
& \bar{w} *(0)=-B_{33} \bar{\tau}_{z z}^{*}(0)
\end{aligned}
$$

Vertical stress comes from the force exerted by the ballast $F_{\mathrm{B}}(x, t)$ on the surface of lateral length $2 L_{\mathrm{BAL}}$. So, displacement is written:

$\bar{w}^{*}(0)=-B_{33} \bar{F}_{\mathrm{B}}(\beta) \frac{\sin \gamma L_{\mathrm{BAL}}}{\gamma L_{\mathrm{BAL}}}$

\subsection{Description of the train load}

Typical train excitation is more complex than that represented previously (Fig. 2). It is composed of a series of vertical excitation, modelling the effect of each engine and carriage wheel on the rail. Each of these forces is considered as harmonic. The total weight of the load is distributed over the whole rail-wheel contact.
Cauchy stress due to the engine is given by (cf. Fig. 3):

$$
\begin{aligned}
\bar{\sigma}_{\mathrm{ENG}}(\beta)= & -2 P_{\mathrm{ENG}} \mathrm{e}^{i Q_{0} t} \mathrm{e}^{-i \frac{\beta}{2}\left(N_{\mathrm{WAG}} L_{\mathrm{WAG}}+L_{\mathrm{ENG}}\right)} \frac{\sin \beta a}{\beta a}[1 \\
& \left.+2 \cos \beta L_{\mathrm{BOG}-\mathrm{ENG}}\right] \cos \beta L_{\mathrm{AX}-\mathrm{ENG}}
\end{aligned}
$$

and the Cauchy stress due to $2 N_{\mathrm{WAG}}$ carriages:

$$
\begin{aligned}
\bar{\sigma}_{\mathrm{WAG}}(\beta)= & -4 P_{\mathrm{WAG}} \mathrm{e}^{i \Omega_{0} t} \frac{\sin \beta a}{\beta a} \cos \beta L_{\mathrm{AX}-\mathrm{ENG}} \\
& \times \cos \beta L_{\mathrm{BOG}-\mathrm{WAG}} \sum_{k=1}^{2 N_{\mathrm{WAG}}} \mathrm{e}^{i \beta\left(k-\left(N_{\mathrm{WAG}} / 2\right)-(1 / 2)\right) L_{\mathrm{WAG}}}
\end{aligned}
$$

where $P_{\mathrm{ENG}}$ and $P_{\mathrm{WAG}}$ are the engine and carriage weight, $N_{\mathrm{WAG}}$ is the number of carriages, $L_{\mathrm{BOG}-\mathrm{ENG}}$ is the length between two engine bogies, $L_{\mathrm{BOG}-\mathrm{WAG}}$ is the length between two carriage bogies, $L_{\mathrm{AX}-\mathrm{ENG}}$ and $L_{\mathrm{AX}-\mathrm{WAG}}$ is the length between two engine and carriage axles.

Finally, for a standard train: $\bar{\sigma}_{\text {TRAIN }}(\beta)=\bar{\sigma}_{\mathrm{ENG}}(\beta)+$ $\bar{\sigma}_{\text {WAG }}(\beta)$.

Excitation of different axles is taken to be synchronous. However, the study of non-synchronous excitation can be carried out by introducing a time delay in Eqs. (31) and (32). Parameters for a real train (the SNCF 'Corail' and 'Autorail') are given in Table 1. In the first example we consider only two carriages (Fig. 3). In the wave number domain the distribution function of the train is a periodical and symmetrical function when the speed is equal to zero. Fig. 3 describes Cauchy stress in the real field and in accordance with Fig. 2. The magnitude of this stress depends on the weight of each axle and the surface area of wheel-rail contact.

\subsection{Numerical results}

Fig. 4 shows vertical displacement on the soil's surface in the wave number domain. A curve in a dotted line can be obtained for a model of a layer lying on a half-space excited by a harmonic rectangular load (by Lefeuve [32]). The resulting curve in the $(\beta, \gamma)$ plane for a 3D model without a track is deduced by a rotation of this previous curve around the $w^{*}$ vertical axle and is a symmetrical function of $\beta$ and $\gamma$. The curve in a full line against $\beta$ associated with the curve in a dotted line against $\gamma$ represents the model with a track and is not a symmetrical function. The presence of the track

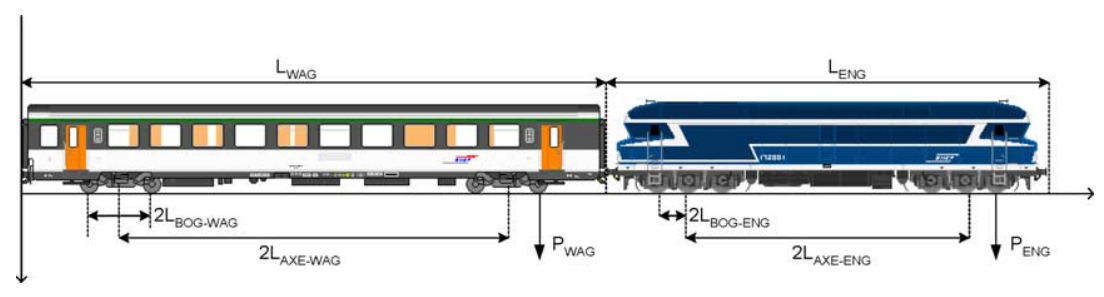

Fig. 2. Representation of train excitation. 

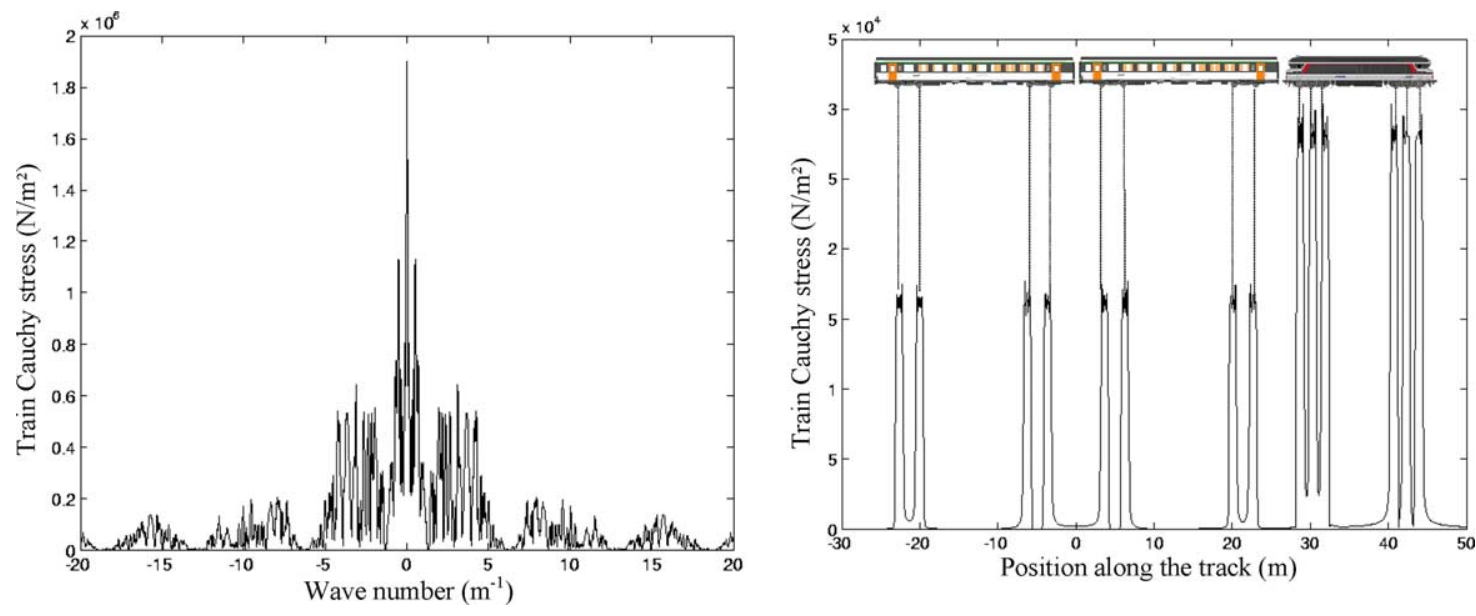

Fig. 3. Cauchy stress in wave number (left) and real fields (right).

Table 1

Parameters of standard trains

\begin{tabular}{lllllll}
\hline & $P_{\mathrm{ENG}}(\mathrm{kN})$ & $P_{\mathrm{WAG}}(\mathrm{kN})$ & $L_{\mathrm{BOG}-\mathrm{ENG}}(\mathrm{m})$ & $L_{\mathrm{BOG}-\mathrm{WAG}}(\mathrm{m})$ & $L_{\mathrm{AX}-\mathrm{ENG}}(\mathrm{m})$ & $L_{\mathrm{AX}-\mathrm{WAG}}(\mathrm{m})$ \\
\hline Autorail & 108 & 78 & 2.5 & 2.5 & 15.2 & 15.4 \\
Corail & 180 & 103 & 1.6 & 2.56 & 11.99 & 16.3 \\
\hline
\end{tabular}

modifies the magnitude of waves emitted in the first layer of the ground and, more precisely, amplifies the displacement in the direction of the track.

Increasing load frequency leads to a reduction in the peak of resonance and this is obtained for higher Mach numbers (Fig. 5). The effect on vertical displacement non-dimensionalised by the Rayleigh wave length is also studied for increasing speeds of harmonic unit load $(40 \mathrm{~Hz})$ moving on semi-infinite ground. For a non-moving load $\left(M_{\mathrm{R}}=0\right)$ or a load moving at sub-Rayleigh speeds $\left(M_{\mathrm{R}}=0.5\right)$, displacement is symmetrical and the maximum coincides with the load position. For super-Rayleigh speeds $\left(M_{\mathrm{R}}=1.5\right)$, the maximum is shifted behind the load position. The critical speed $\left(M_{\mathrm{R}}=1\right)$ between these two regimes amplifies displacement.

Now, the final track model (train-track-soil) discussed in paragraph 2.1 is used. The ballast is considered as the first layer of the ground (thickness $h_{\mathrm{BAL}}$ ) and interaction is obtained by sleepers (width $2 L_{\text {TRAV }}$ ). Fig. 6 shows a comparison between displacement below the track in the real field for each speed regime (for a $10 \mathrm{~Hz}$ unit load). Mechanical parameters correspond to soft peaty ground (Table 2). If the load speed is lower than the phase speed of the system, displacement produced by each train force decreases quickly and is limited to the vicinity of this force. However, if the speed load increases enough to excite a propagation mode in the system, there is amplification due to each force response.

An increase in speed thus implies an amplification of vertical displacement and an amplification of oscillation behind the train. Displacement field on the surface of the ground is represented in Fig. 6 for different speeds and confirms previous results as well as the presence of Mach cones for each bogie (Fig. 7).

At the end of this theoretical study of railway vehicle vibration, numerical software called FASTIVIB was built. 'Fourier Analysis of Soil-Track Interaction and VIBration' is a software programme that evaluates displacement on the surface of homogeneous and isotropic semi-infinite or multilayered soil. It takes into account the physical characteristics of the soil under a railway track and a simplified model of a train moving at constant speed.

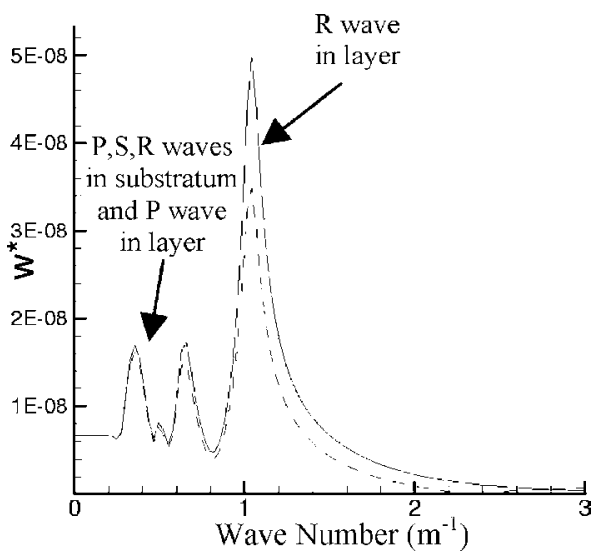

Fig. 4. Vertical displacement in the Fourier domain following $\beta(-)$ and $\gamma$ $(-)$ axes. 

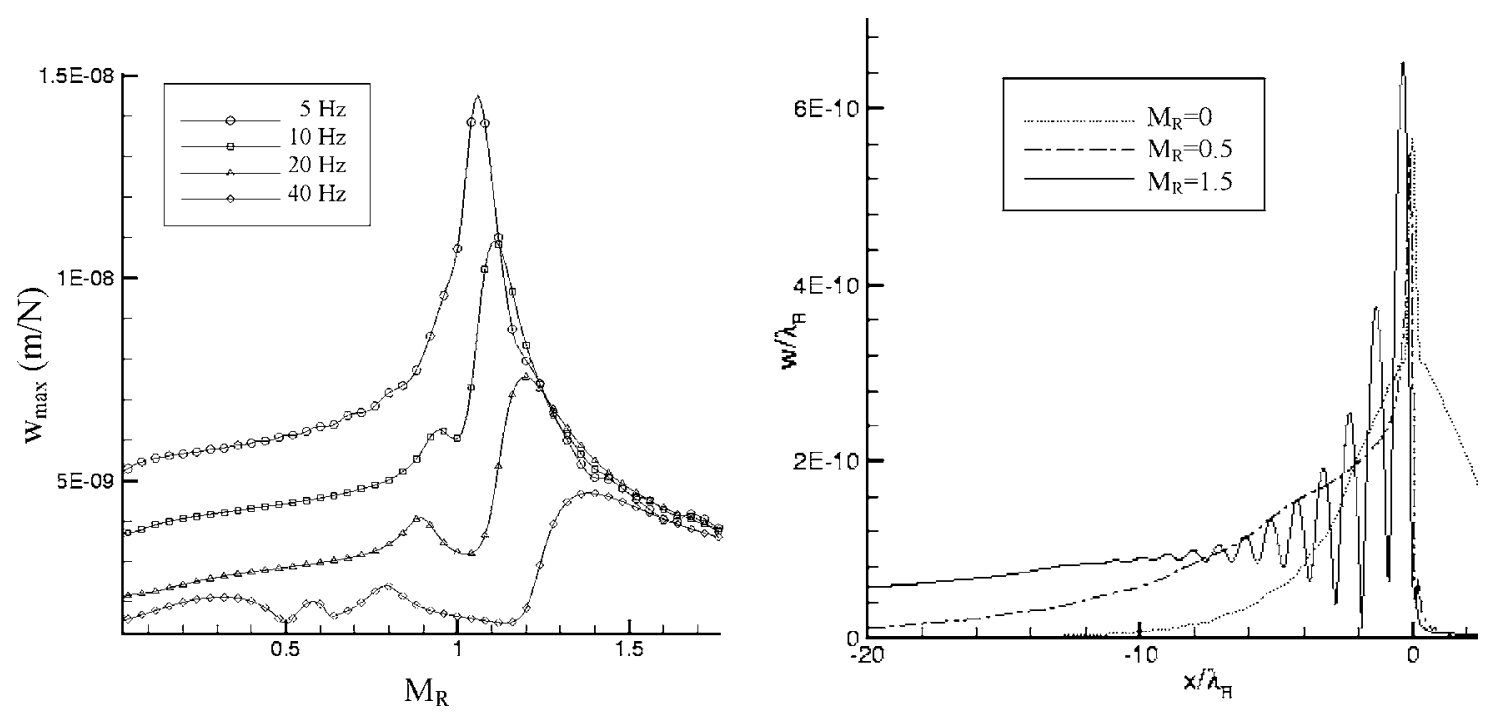

Fig. 5. Critical speed for moving load on soft soil (left) and vertical displacement against the load speed (right).

The mathematical track model in FASTIVIB is obtained with the following simplifications:

- each rail is considered as perfectly elastic (Euler beam),

- each sleeper is modelled by a sum of continuous stiffness elements connected to the rail and allowing a longitudinal and lateral strength,

- railpads are considered as stiffness and damping elements between the sleepers and the rail,

- the ballast can be constituted either simply with vertical stiffness elements allowing a longitudinal and lateral rigidity of the structure, or as an elastic layer lying on the ground's surface.

\section{Diagnosis of vibration from in-situ measurements}

\subsection{Location and soil properties}

Special sites with high levels of displacement are found in the North West of France (near Amiens) along the railway

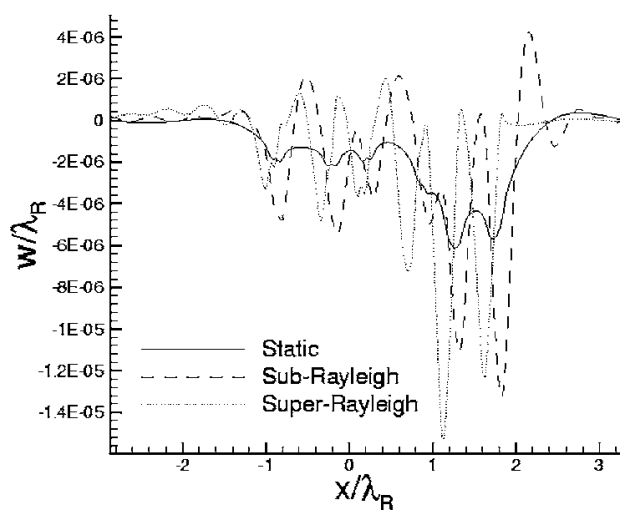

Fig. 6. Effect of train speed on the vertical displacement under the track. line from Longueau to Boulogne. Two tracks are concerned where the ground is composed mainly of alluvia (clay mixed with peat-low Young modulus $E$ ) or silt often found along the valley of the river Somme.

The studied area is located at the South West boundary of this valley. It is characterised by a very thick alluvium layer lying directly on a limestone substratum. The density $\rho$ of the ground can easily be measured. It is determined using borings in the vicinity of the track. A sample (size: depth $7 \mathrm{~m}$, diameter $70 \mathrm{~mm}$ ) has been analysed. For different sections normal and hydrostatic weighing tests are performed successively. To identify the Young modulus and the Poisson ratio, $P, S$ and $R$ wave speeds are obtained from seismic refraction tests.

Compression and shear wave speeds are connected to parameters of the behavior law of soil by:

$c_{\mathrm{P}}=\sqrt{\frac{\lambda+2 \mu}{\rho}} c_{\mathrm{S}}=\sqrt{\frac{\mu}{\rho}}$

Table 2

Mechanical parameters for the two sites concerned

\begin{tabular}{lllllll}
\hline BREILLY & & & & & & \\
\hline & $\begin{array}{l}C_{\mathrm{p}} \\
(\mathrm{m} / \mathrm{s})\end{array}$ & $\begin{array}{l}C_{\mathrm{s}} \\
(\mathrm{m} / \mathrm{s})\end{array}$ & $\begin{array}{l}E \\
(\mathrm{MPa})\end{array}$ & $\nu$ & $\begin{array}{l}\rho(\mathrm{kg} / \\
\left.\mathrm{m}^{3}\right)\end{array}$ & $\eta$ \\
\hline Peat $(3 \mathrm{~m})$ & 344 & 147 & 96 & 0.39 & 1600 & 0.05 \\
Transition $(4 \mathrm{~m})$ & 960 & 480 & 940 & 0.37 & 1850 & 0.08 \\
Limestone ( $\infty)$ & 1872 & 930 & 4539 & 0.34 & 2000 & 0.1 \\
CROUY-ST-PIERRE & & & & & \\
& $C_{\mathrm{p}}(\mathrm{m} /$ & $C_{\mathrm{s}}(\mathrm{m} /$ & $E$ & $\nu$ & $\rho(\mathrm{kg} /$ & $\eta$ \\
& $\mathrm{s})$ & $\mathrm{s})$ & $(\mathrm{MPa})$ & & $\left.\mathrm{m}^{3}\right)$ & \\
Peat $(3 \mathrm{~m})$ & 345 & 122 & 68 & 0.43 & 1600 & 0.05 \\
Transition $(4 \mathrm{~m})$ & 959 & 475 & 940 & 0.37 & 1850 & 0.08 \\
Limestone $(\infty)$ & 1850 & 920 & 3889 & 0.35 & 2000 & 0.1 \\
\hline
\end{tabular}



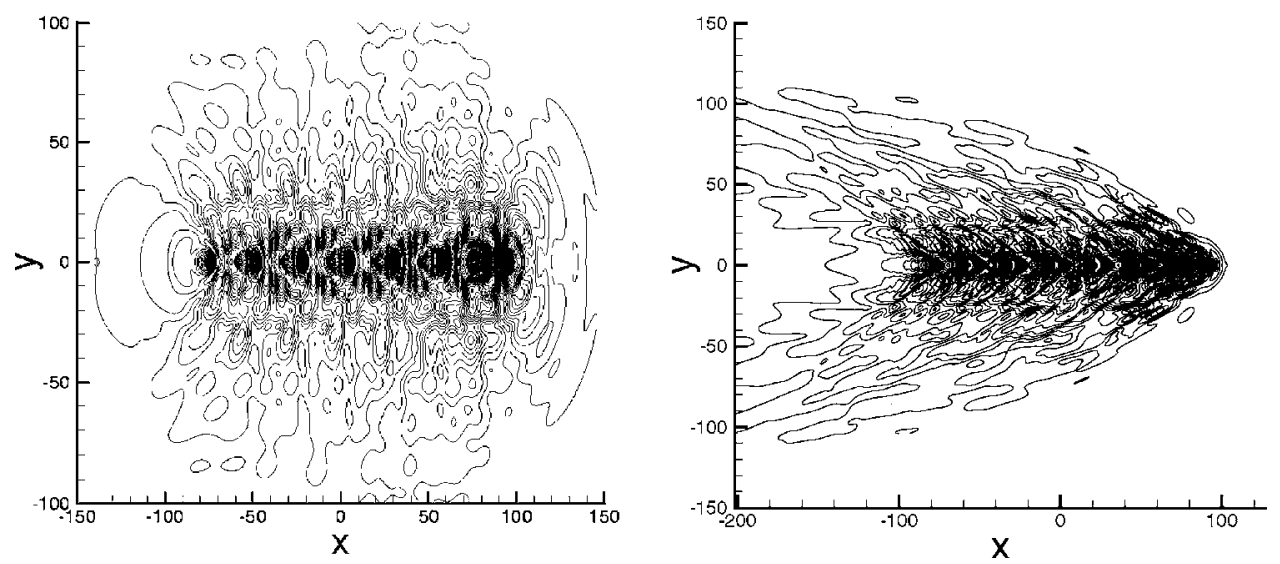

Fig. 7. Displacement field on soil surface for Corail train in sub (left) and super Rayleigh (right) regimes.

As Lamé coefficients $\lambda$ and $\mu$ are joined to Young modulus $E$ and to Poisson ratio $\nu$, these mechanical parameters can be deduced as follows:

$E=\rho c_{\mathrm{S}}^{2} \frac{3\left(\frac{c_{\mathrm{P}}}{c_{\mathrm{S}}}\right)^{2}-4}{\left(\frac{c_{\mathrm{p}}}{c_{\mathrm{S}}}\right)^{2}-1} \quad \nu=\frac{1}{2} \frac{\left(\frac{c_{\mathrm{p}}}{c_{\mathrm{S}}}\right)^{2}-2}{\left(\frac{c_{\mathrm{p}}}{c_{\mathrm{S}}}\right)^{2}-1}$

The damping ratio $\eta$ characterising the material is identified by the evolution of the frequency relationship between two spectral measurements at two different points. Datoussaid et al. in [33] have shown that the mean of this curve allows us to deduce the increase in damping with frequency.

Finally, Table 2 sums up the main results in two locations: 'Breilly' and 'Crouy St Pierre'. The simulation model is built with the data in this table. It appears that the two sites are slightly different in terms of Young modulus and Poisson ratio. Density and damping ratio are assumed to be the same.

\subsection{Measurements network}

Numerical camcorder CCD (MotionVision) of spacial resolution $528 \times 512$ pixels, containing an acquisition card coupled with unit software (SYSMAT Industry VNR250) is used. With this process, we can evaluate longitudinal and vertical displacement of the rail as well as deformation using four marks painted on the rail [34]. The frequency of acquisition is equal to 250 images per second. In this case, the time of acquisition is close to $7 \mathrm{~s}$ (approximately 1800 images). Analysis of recordings is carried out in deferred time thanks to a program that automatically calculates the displacement for each image between the marks and their positions. The centre is located by calculating the barycentre balanced by bright colours. The precision of measurement is dependent on the size of the mark. Finally, local plane displacement of the rail is easily deduced from these marks. In addition,
Laser velocimeter (RMP-POLYTEC OFV 2200) aiming at a reflection cell glued to the rail for lateral velocity measurements is also used.

An accelerometer BandK type 4371 is magnetised on the rail for vertical measurements and on the flank of the rail for lateral measurements. It is subjected to very high acceleration as well as high noise levels propagated by the rail. One accelerometer (ICP 393A03) is glued to the surface of the sleeper for vertical measurements or on its side for lateral measurements and the other one is fixed to a steel support sunk into the ground in different parallel and perpendicular points to the track.

The technique of a trigger starting with infrared detection enables automatically analysis of intersection of the infrared ray by the passage of the train. The speed of trains then can be calculated precisely.

Displacement of each part of the track and of ground surface is calculated with sufficient precision from acceleration signals measured by accelerometers. However, particular care must be taken during data processing of transducers used to record acceleration and to recording parameters such as the sampling frequency. Thanks to the asynchronous recording of numerical and analogical data, a sampling frequency of $1000 \mathrm{~Hz}$ is used (compared to $250 \mathrm{~Hz}$ for the camera) to obtain a sufficiently accurate displacement calculation. Thus, measured acceleration signals are integrated to deduce speeds and displacement by using one or two numerical integrations.

\subsection{Experimental results}

Figs. 8 and 9 show absolute rail displacement when Corail and Autorail trains are moving on the track. In Fig. 8, with the same speed $(120 \mathrm{~km} / \mathrm{h})$, the Corail train is composed of one or two engines. In Fig. 9, the Autorail train pulls two or four carriages at a speed equal to $75 \mathrm{~km} / \mathrm{h}$. 

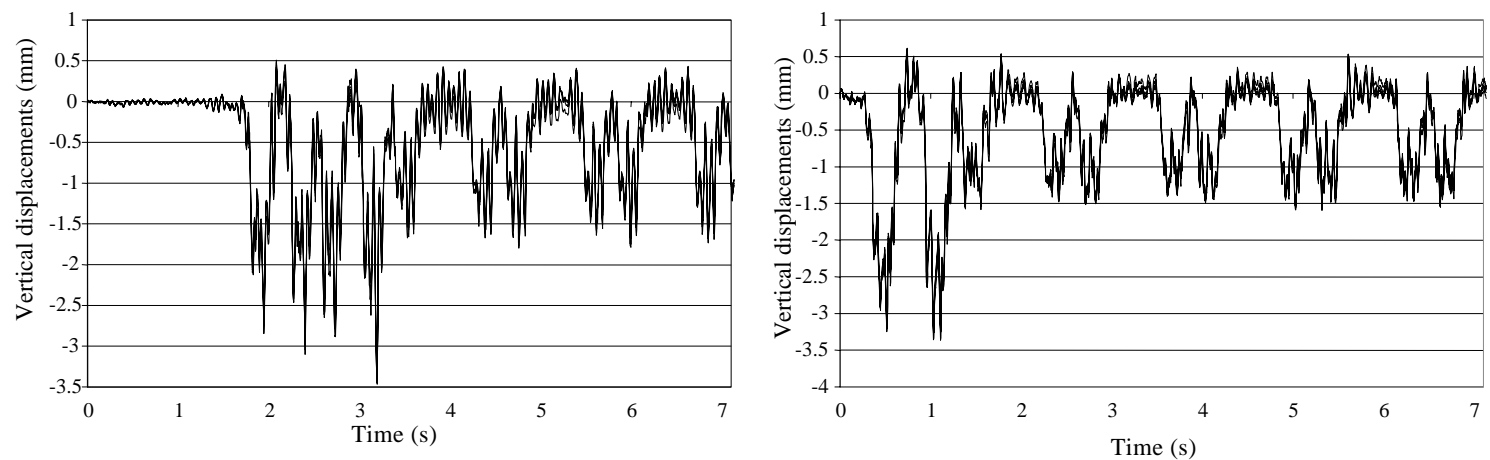

Fig. 8. Data from VNR for Corail train with 2 (left) and 1 (left) engines $(120 \mathrm{~km} / \mathrm{h})$.
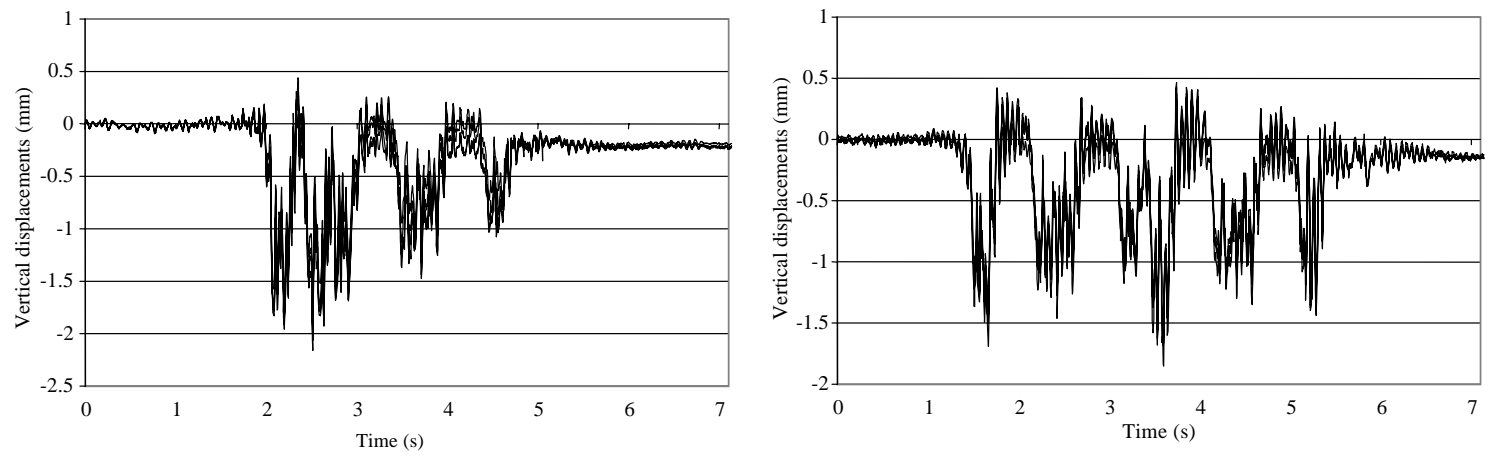

Fig. 9. Data from VNR for short-2 carriages-(left) and long-4 carriages-(right) Autorail trains $(75 \mathrm{~km} / \mathrm{h}$ ).

Each train element can be discerned and the noise signal is relatively low. For these speeds, rail deformation and consequently ground displacement is mainly quasi-static. The maximum displacement amplitude is about $4 \mathrm{~mm}$ for a load equal to $2.10^{5} \mathrm{kN}$ by axle. Fig. 10 shows the rail displacement at a speed equal to $75 \mathrm{~km} / \mathrm{h}$ for a freight train (two engines and twenty two carriages) and only two engines. Measurements of acceleration in the parts of the track (rail, sleepers, pads) are carried out for each passage. The peaks of displacement and maximum particle speeds on the site are compared for a Corail and Autorail train and are summed up in Table 3.
Fig. 11 represents acceleration signals for a train and the displacement spectra calculated by double integration and Fourier transform. The response spectra are calculated using the sampling frequency of $F s=$ $1000 \mathrm{~Hz}$ and a number of points $N=8192$ points. The frequency $f$ and the spectra $S_{\mathrm{w}}$ are then expressed respectively as:

$f=F s \frac{M}{N}$ and $S_{\mathrm{w}}=\frac{F F T(w)}{F s}$

where $M$ varies from 0 to the Nyquist frequency and FFT denotes the Fast Fourier Transform.
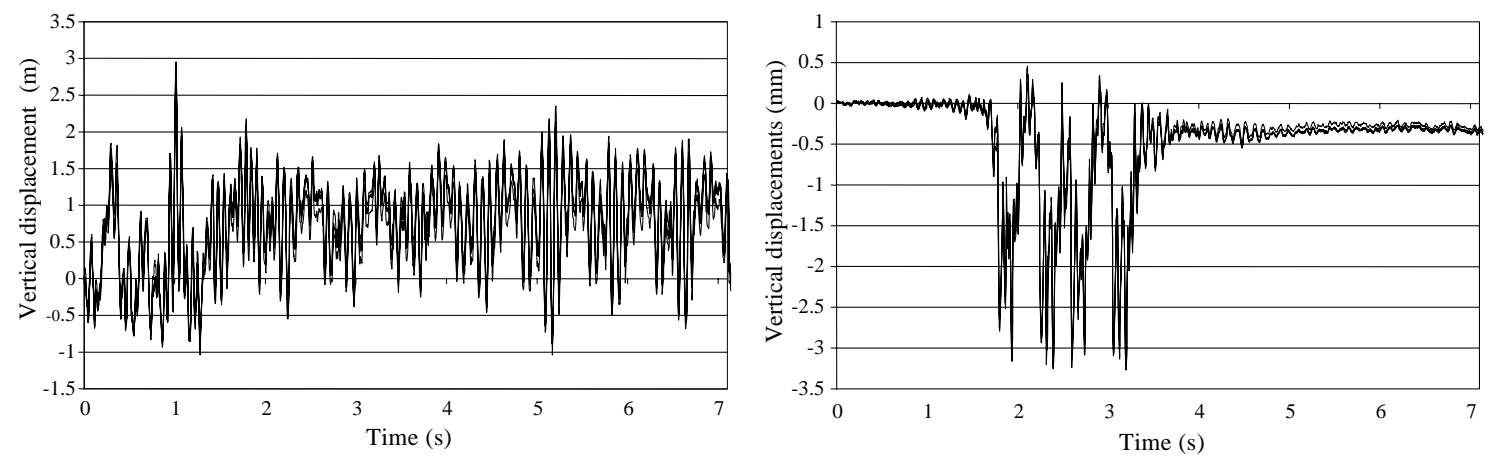

Fig. 10. Data VNR for a freight train—-two engines and 22 carriages (left) and two engines (right). 
Table 3

Particle speeds and maximum displacement

\begin{tabular}{|c|c|c|c|c|}
\hline \multirow[b]{2}{*}{ Corail } & \multicolumn{2}{|c|}{ Particle speed $(\mathrm{mm} / \mathrm{s})$} & \multicolumn{2}{|c|}{ Induced displacement (mm) } \\
\hline & Engine & Carriages & Engine & Carriages \\
\hline Sleeper & - & - & - & - \\
\hline Ballast & 120 & 40 & 5 & 2 \\
\hline Soil at $2 \mathrm{~m}$ & 60 & 40 & 3 & 0.6 \\
\hline Soil at $3 \mathrm{~m}$ & 40 & 1 & 1.4 & 0.4 \\
\hline Autorail & & & & \\
\hline Sleeper & 600 & - & - & - \\
\hline Ballast & 100 & - & 0.8 & 0.3 \\
\hline Soil at $2 \mathrm{~m}$ & 40 & 0.5 & 1.6 & 0.2 \\
\hline Soil at $3 \mathrm{~m}$ & 20 & 0.25 & 0.8 & 0.1 \\
\hline
\end{tabular}

Signals are the same with an attenuation factor in amplitude close to 10 . Note that the signal measured in theballast can be strongly faulty due to the unsatisfactory interaction between granular medium and
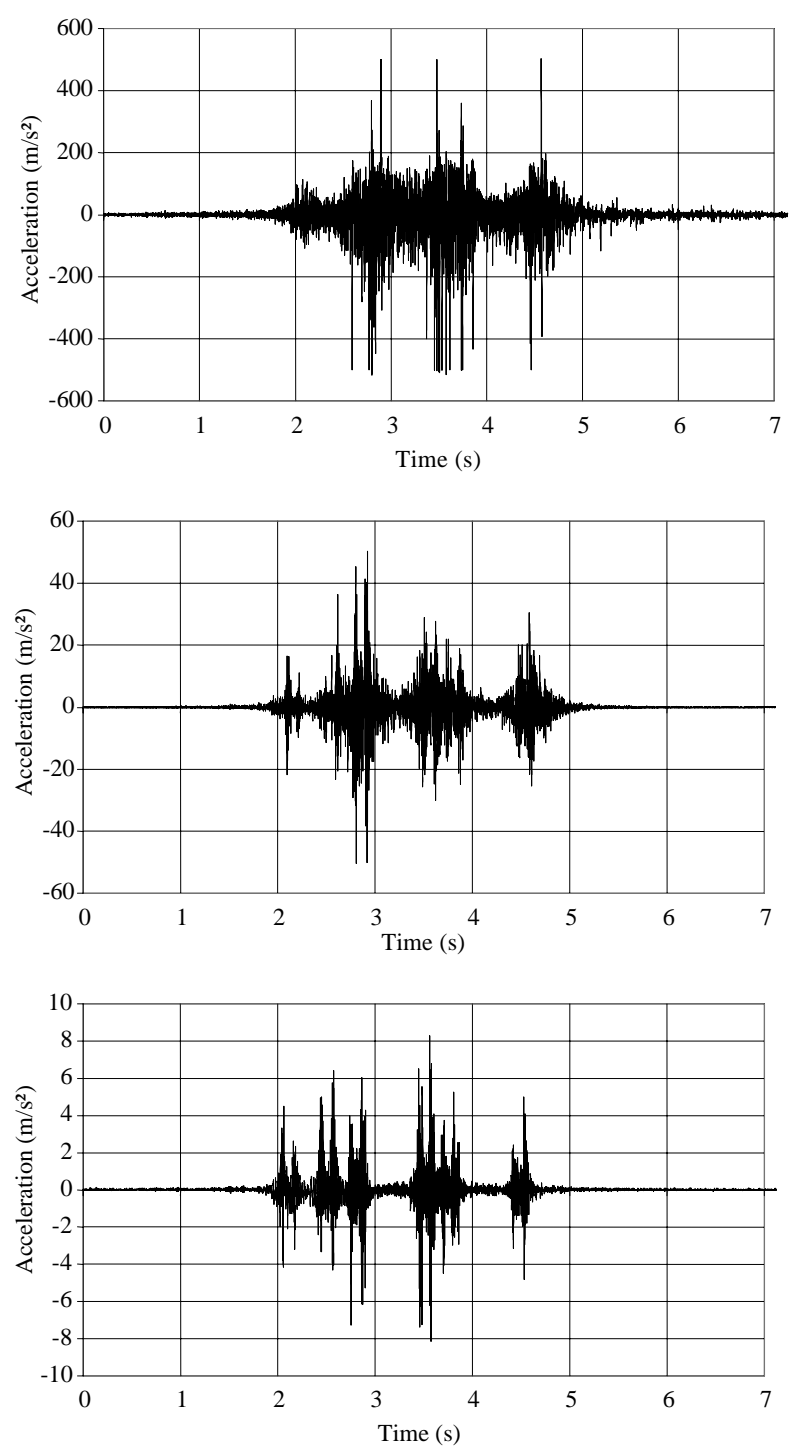

the accelerometer. The spectra evolve from one element to the other and we find that the highest peaks in sleepers and in the ballast are due to the passage of axles. In the 10 $20 \mathrm{~Hz}$ range, amplitude is smaller.

Fig. 12 gives results for surface measurements of the ground near the track $(2,3$ and $5 \mathrm{~m})$ for the same passage and train speed (Autorail train moving at $80 \mathrm{~km} / \mathrm{h}$ ). The wave propagation in the medium is necessarily taken into account to perform measurements far from the track because very high quasi-static deflections mask vibration. These high deflections are visible on the spectra. For example, near the track, a high peak between 2 and $5 \mathrm{~Hz}$ masks vibration (in the time domain) with consequences on components from 10 to $40 \mathrm{~Hz}$. These deflections are not as visible at 3 and $5 \mathrm{~m}$. The acceleration signal measured at $5 \mathrm{~m}$ is slightly higher than the result at $3 \mathrm{~m}$. But these results wholly depend on the relation between the wavelength and the
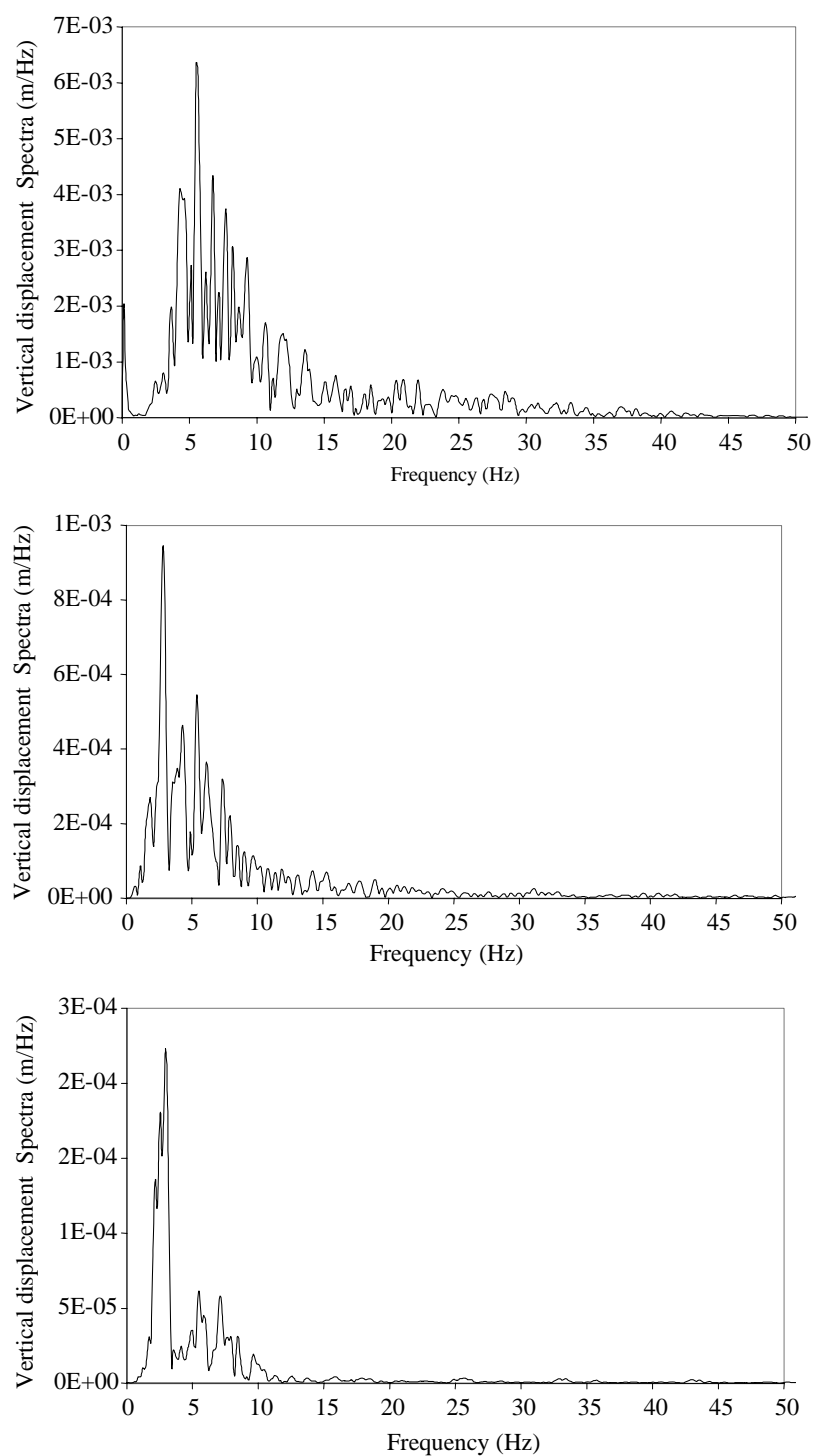

Fig. 11. Measurements on a track for a train moving at $75 \mathrm{~km} / \mathrm{h}$ (from top to bottom: rail, sleeper, ballast). 

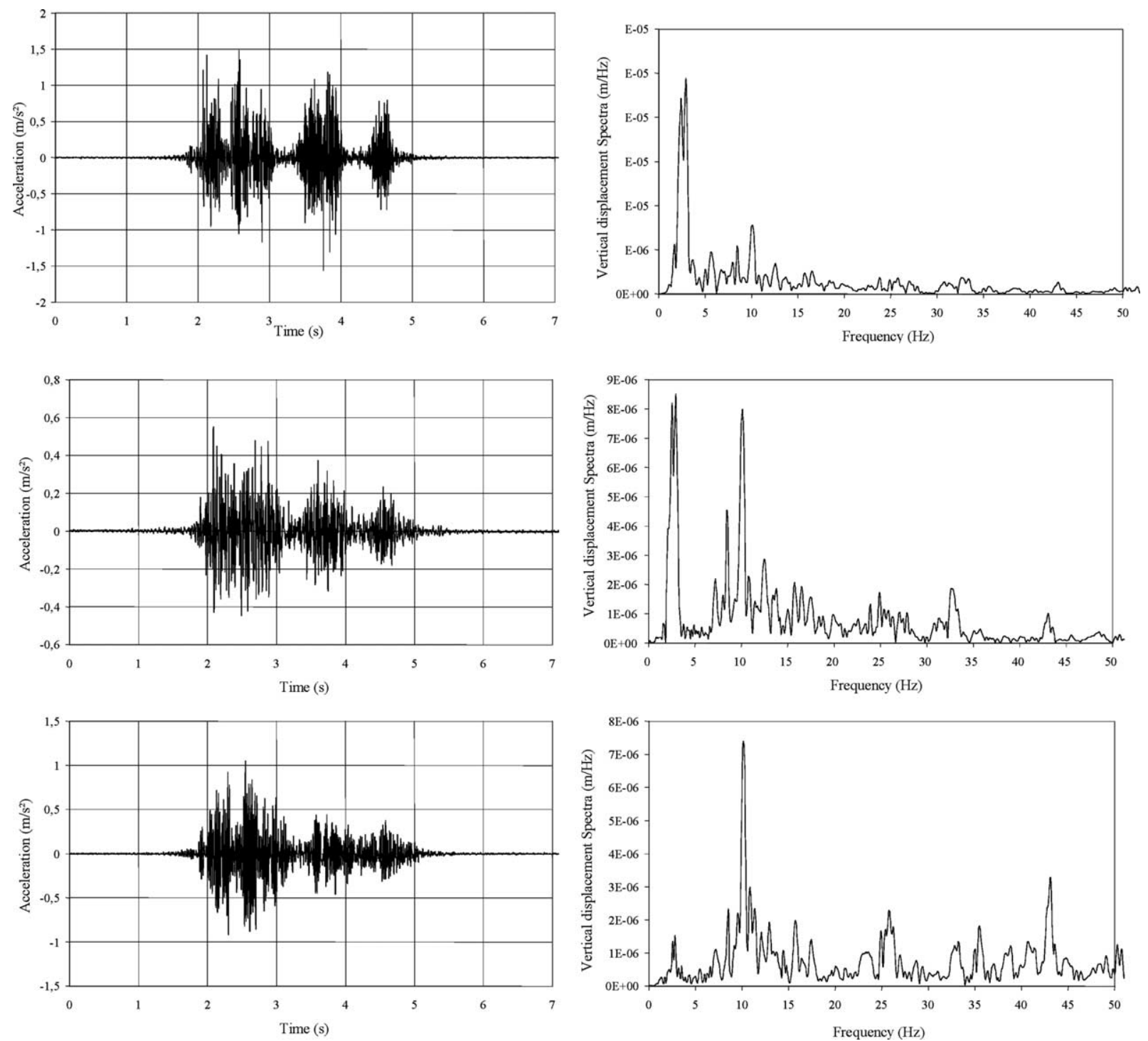

Fig. 12. Measurements on soil surface for a train moving at $75 \mathrm{~km} / \mathrm{h}$ (from top to bottom: $2,3,5 \mathrm{~m}$ ).

recording position chosen for the experimental measurement.

Fifteen passages including various parameters such as the train type, its speed, the site characteristics are recorded by Picoux et al. and summarised in [34].

The study of spectral acceleration density shows that:

- vertical accelerations is very much reduced by the ballast,

- their spectra distribution is different from one element to another,

- on a same site, a heavy train (Corail) carries higher acceleration in the $50-100 \mathrm{~Hz}$ range than a freight train and an Autorail train,

- for the highest frequencies, a gap is fully perceptible between the modified and the nonmodified site.

\section{Validation of the model and use in prediction}

\subsection{Parametric analysis}

A '2-layer' model is made up of a ballast $(0,8 \mathrm{~m})$ and a peat layer $(7 \mathrm{~m})$ lying on a limestone substratum. The maximal amplitude of the velocity signal is represented as a function of the Young modulus in Fig. 13. We can observe that the maximal amplitude of the signal increases when the frequency of excitation and the Young modulus of the peat decrease. In the range of usual Young modulus for the considered site (50-100 MPa), the particle speed of the soil surface is less sensitive to the excitation frequency than for lower modulus. This representation will hereafter allow us to approach and to calibrate the model using measurements.

In the case of linear approximation, the principle of superposition can be applied for a study including 


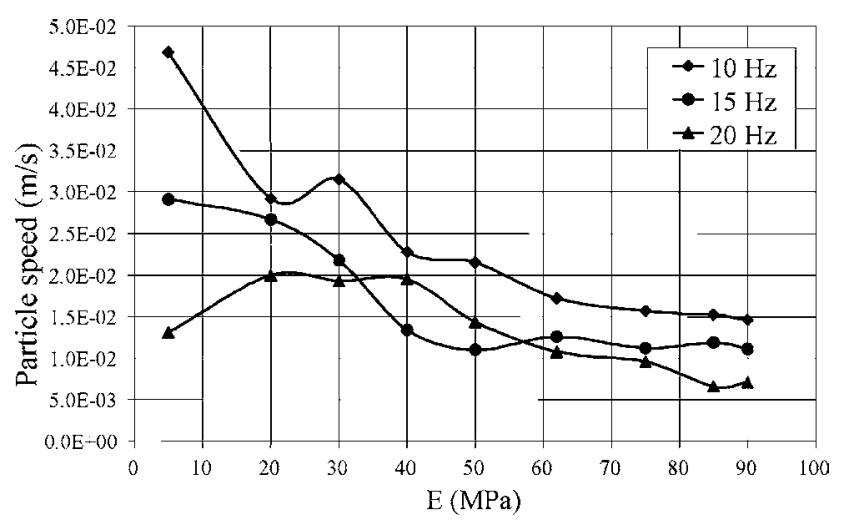

Fig. 13. Influence of Young modulus on maximal particle speed.

several frequencies. An example is given in Fig. 14 in the wave number domain and in the real field for a harmonic rectangular load. Supplementary peaks corresponding to excitation frequencies can be noted as well as a change in shape of the temporal signal and the amplitude under the load. In addition to the response superposition, the weight function depends on a frequency between 0 and $80 \mathrm{~Hz}$.
While it has already been previously noted in the framework of a multifrequential study, the total excitation includes a quasi-static component (weight of train axles) and a harmonic component (irregularities due to wheel-rail contact). The excitation is therefore a function of the frequency. Near the track, static and quasi-static responses are preponderant. The model used for comparisons at 2 or $3 \mathrm{~m}$ includes the contribution of the constant component and of a low harmonic frequency component. Below $5 \mathrm{~m}$, the contribution of the harmonic component becomes more important.

\subsection{Comparison between simulation results and measure- ment data}

For this comparison, the spectral densities of displacement show a large discrepancy in the range $0-20 \mathrm{~Hz}$ and particle speeds are not easily comparable, certainly due to experimental conditions and perhaps a less accurate model used for the multifrequential representation of Autorail train excitation. Similar difficulties have been noted by Paolucci et al. [25] for the Ledsgaard site (Sweden). Fig. 15 shows signals measured in situ
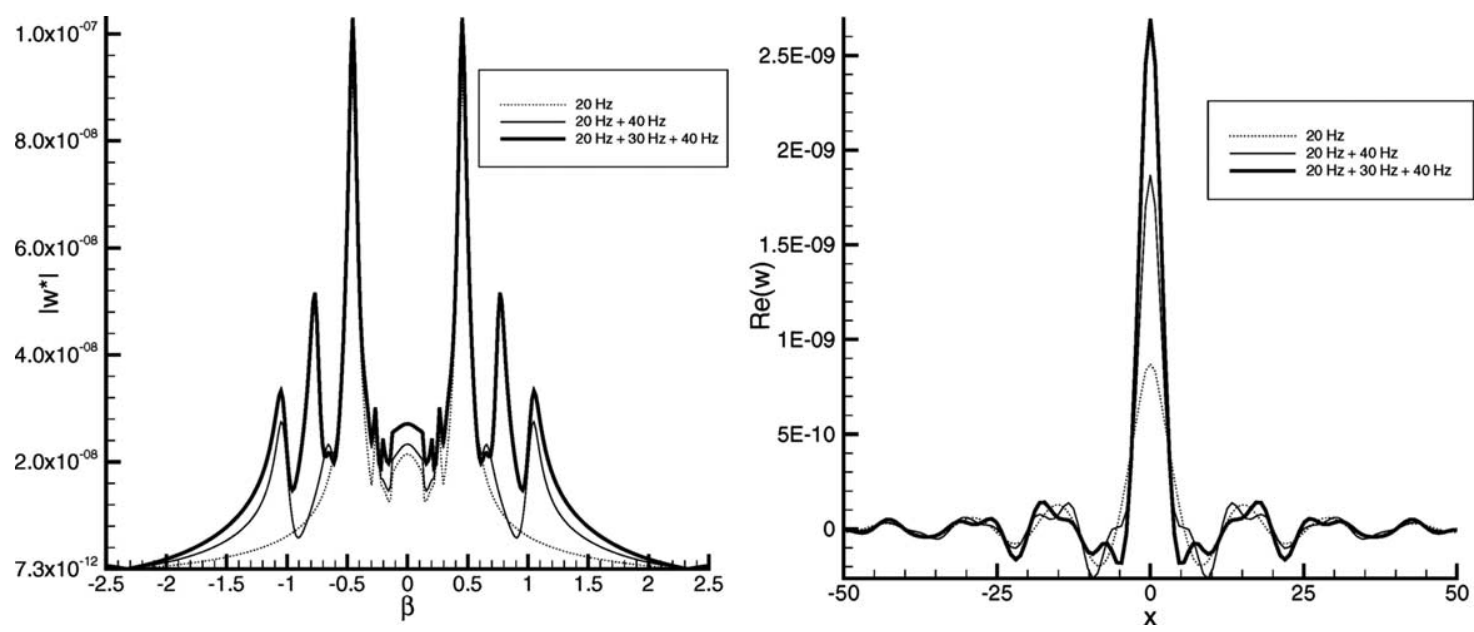

Fig. 14. Frequency combination in the Fourier domain and in real field.
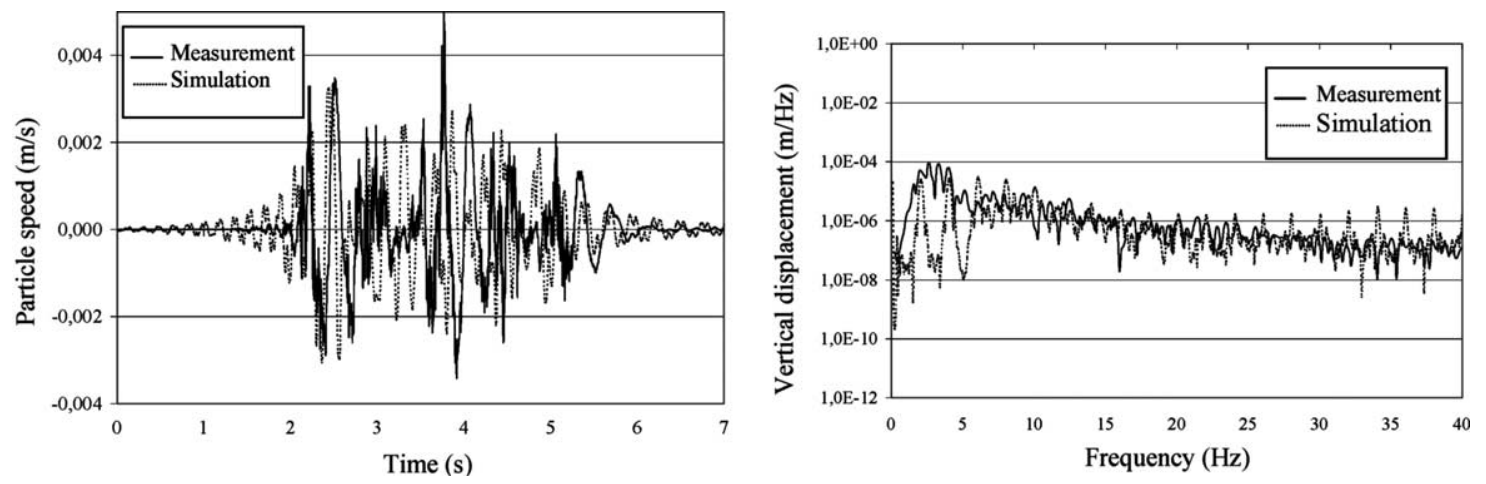

Fig. 15. Comparison for Autorail train: particle speed at $2 \mathrm{~m}$ from track (left), spectral density of displacement (right). 

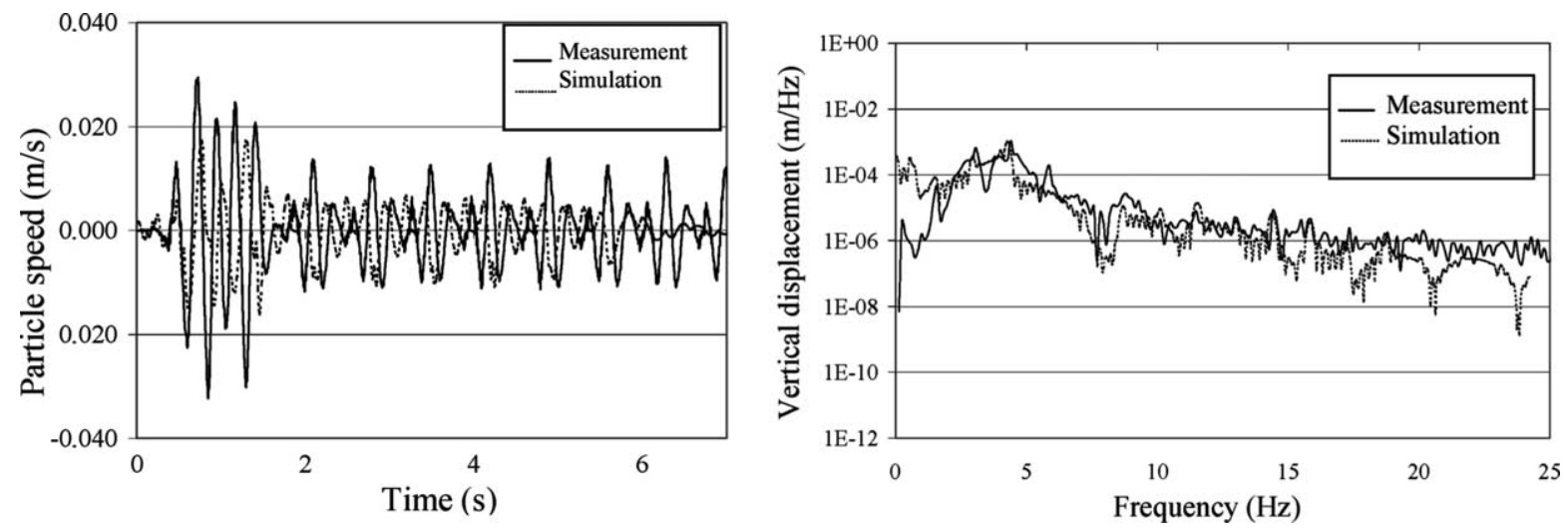

Fig. 16. Comparison for Corail train: particle speed at $2 \mathrm{~m}$ from track (left),spectral density of displacement (right).

and calculated at $2 \mathrm{~m}$ from the track during the passage of a light train (Autorail). The spectral density of vertical displacement calculated with a complete model between 0 and $80 \mathrm{~Hz}$ can then be compared to the measurement data from near the track.

Finally, Fig. 16 introduces results for a Corail train (two engines) moving at a higher speed $(135 \mathrm{~km} / \mathrm{h})$. In this case, the simulation model gives results that are in better accordance with spectral density and particle speeds. Note that for this calculation, a train made up of six carriages was studied while for the measurements, the train had 12 carriages. This point can explain after $6 \mathrm{~s}$ a bigger decrease in the simulation curve than in the measurement curve. Fig. 17 gives us information about the calculated signal according to the distance from the track and shows the quasi-static shape of the surface displacement near the track. This shape disappears the further it gets from the track and gives a harmonic signal.

Farther from the source, the contribution of the harmonic regime due to irregularities becomes more important. The comparison between measurements and results deduced from the numerical simulation according to the distance from the track is also performed. Accordance between the two sets of results confirms the accurate choice of mechanical parameters before fitting using a minimisation process, in particular for those linked to compression and shear wave speeds.

\subsection{Further developments}

An analytical or semi-analytical approach using Fourier transform is therefore suitable for the case of a semi-infinite soil medium. Moreover, it can be used in the case of horizontal homogeneous ground layers. But this method is not possible if the ground includes various heterogeneities (inclusions, tunnels, ...) or buried structures with very different material characteristics. In this case, other more convenient approaches are necessary, for example a numerical model developed by Laghrouche in [35] can be used. This model has been compared with the semi-analytical approach for a semi infinite medium by Lefeuve-Mesgouez et al. in [36] and can be used in future developments to study solutions to specific vibration problems such as: sites with trenches, inclusion of damping layers or the presence of concrete columns under or near the track. Also in our approach, the ground is modelled as an isotropic and homogeneous linear elastic medium with
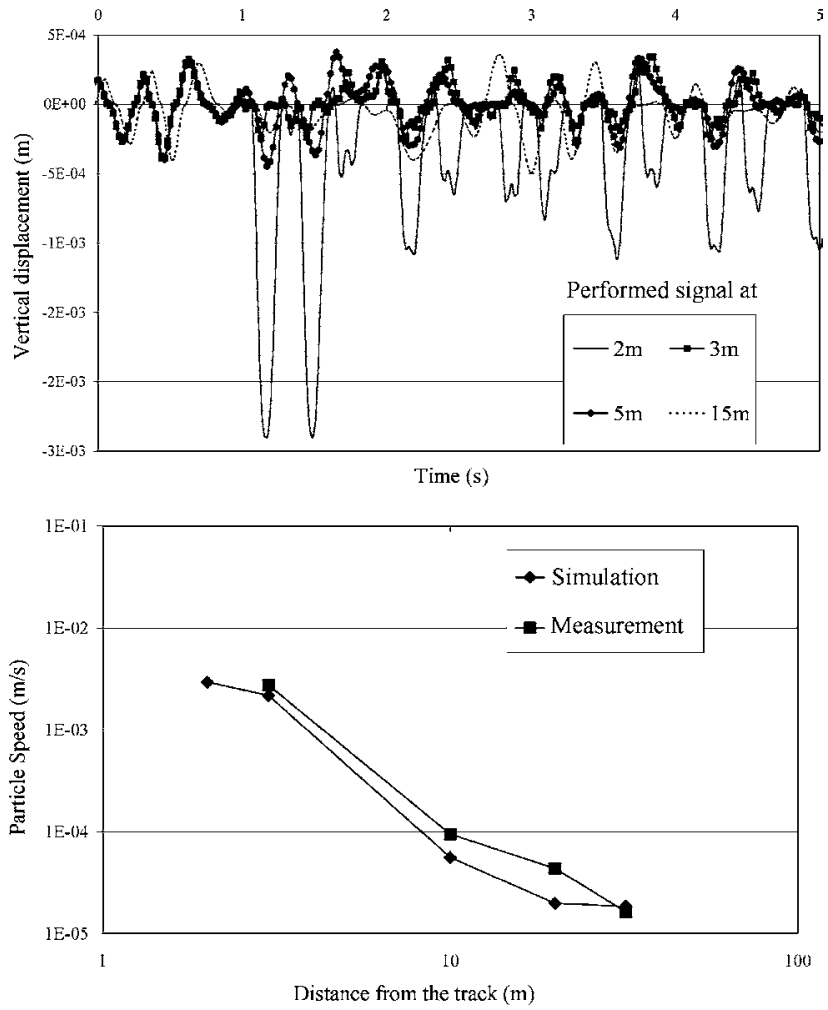

Fig. 17. Distance from the track; top: displacement response against distance, bottom: comparison between simulation and measurements. 
a material dissipation by means of a hysteretic damping hypothesis. To consider the real behaviour of the soil, this model can be improved and for example, the effect of fully saturated poroelastic soil taking into account soil porosity and permeability, could be studied as in [37] and [38] to give a better definition of the organic peat layer. In the same outline, we could study the influence of seasonal variations of the ground water table on rail traffic-induced vibrations, in the same way as [39] for the passage of heavy trucks on a uneven surface. The modelisation of traffic loads also needs supplementary study. The train axle indeed includes a lot of 'massspring' systems between the proper train and the contact on the rail, and for the study, our model is very much simplified. Finally, more accurate data on the contact between wheel and rail would allow better precision in the real frequency range and thus improve the source function approached during the validation.

\section{Conclusions}

The first part of this paper, is concerned with numerical simulation tools suited to the problem of the propagation of vibration from railway traffic and taking into account the various elements of the structure (railtrack-ground). The numerical model is proposed to ensure the interaction between track and ground and possible simplifications for the ground (semi-infinite or multilayered) with, nevertheless, the restriction of a linear hypothesis for the behavior of all materials. The model can be applied to $2 \mathrm{D}$ or $3 \mathrm{D}$ configurations. The adaptation of a fitted argument (FASTIVIB software) has allowed a gain in calculation time (reduction of 50 per cent for a soil including five layers). Despite uncertain geomechanical data, the model has allowed us to explain the relative influence of the various track components. The model enables all possible parametric studies (with or without track, characterisation of all horizontal and vertical displacement in the wave number and real fields).

The use of these models and the validity of the results are directly connected to the accuracy of geomechanical data concerning both the track and more particularly the ground. Consequently, the second part of this paper develops in situ measurements of soft soil. The Rayleigh wave speed in the peat layer is obtained in the $90-110 \mathrm{~m} / \mathrm{s}$ range $(320-400 \mathrm{~km} / \mathrm{h})$. Thus, with a maximal train speed measured at $135 \mathrm{~km} / \mathrm{h}$, the super-Rayleigh regime cannot be reached (Mach number close to 0.4). Despite these conditions, the effect of train speed can be perceived upon observation of trains moving at 70 and $135 \mathrm{~km} / \mathrm{h}$ and the effect of train type has also been observed. Using the average of vertical displacement and by choosing a reference of 1 for an Autorail train, results of approximately 1.7 are obtained for a Corail train and 2.7 for a freight train. The measurement network and the signal analysis have allowed to accurately deduce displacement of each part of the track and on the surface of ground; even if some results are difficult to use (superposition of noise and signal), the influence of several parameters concerning with the railway track can be performed correctly.

Finally, the last part is concerned with the comparison of the numerical results and experimental data. For this operation, it was necessary first to choose suitable data to perform numerical simulations. Parameters of the ground are deduced from seismic measures and borings. Parameters for the track are provided from the S.N.C.F. A problem arose from simulation studies concerning the choice of the excitation frequency. Several models of excitation were proposed (mono-frequential and multifrequential with frequencies in the $5-80 \mathrm{~Hz}$ range). This choice could explain some differences (sometimes large in the case of some frequency domains and trains) obtained between experimental and numerical results. However in many cases, the comparison performed by Picoux in [40] leads us to an acceptable agreement taking into account different uncertainties present in the two approaches. This agreement is sufficiently significant to estimate that the proposed model allows us to obtain suitable information about the behaviour of the track and the surrounding ground.

\section{Appendix}

Displacement matrix $[Q]$

$[Q]$

$$
\left[\begin{array}{cccccc}
\frac{-i \beta \mathrm{e}^{-\alpha_{\mathrm{P}} h}}{\Delta_{\mathrm{P}}} & \frac{-i \beta}{\Delta_{\mathrm{P}}} & \mathrm{e}^{-\alpha_{\mathrm{S}} h} & 0 & 1 & 0 \\
\frac{-i \gamma \mathrm{e}^{-\alpha_{\mathrm{P}} h}}{\Delta_{\mathrm{P}}} & \frac{-i \gamma}{\Delta_{\mathrm{P}}} & 0 & \mathrm{e}^{-\alpha_{\mathrm{S}} h} & 0 & 1 \\
\frac{-i \alpha_{\mathrm{P}} \mathrm{e}^{-\alpha_{1} h}}{\Delta_{\mathrm{P}}} & \frac{i \alpha_{\mathrm{P}}}{\Delta_{\mathrm{P}}} & \frac{\beta \mathrm{e}^{-\alpha_{\mathrm{S}} h}}{\alpha_{\mathrm{S}}} & \frac{\gamma \mathrm{e}^{-\alpha_{\mathrm{S}} h}}{\alpha_{\mathrm{S}}} & -\frac{\beta}{\alpha_{\mathrm{S}}} & -\frac{\gamma}{\alpha_{\mathrm{S}}} \\
\frac{-i \beta}{\Delta_{\mathrm{P}}} & \frac{-i \beta \mathrm{e}^{-\alpha_{\mathrm{P}} h}}{\Delta_{\mathrm{P}}} & 1 & 0 & \mathrm{e}^{-\alpha_{\mathrm{S}} z} & 0 \\
\frac{-i \gamma}{\Delta_{\mathrm{P}}} & \frac{-i \gamma \mathrm{e}^{-\alpha_{\mathrm{p}} h}}{\Delta_{\mathrm{P}}} & 0 & 1 & 0 & \mathrm{e}^{-\alpha_{\mathrm{S}} h} \\
\frac{-i \alpha_{\mathrm{P}}}{\Delta_{\mathrm{P}}} & \frac{i \alpha_{\mathrm{P}} \mathrm{e}^{-\alpha_{\mathrm{P}} h}}{\Delta_{\mathrm{P}}} & \frac{\beta}{\alpha_{\mathrm{S}}} & \frac{\gamma}{\alpha_{\mathrm{S}}} & \frac{-\beta \mathrm{e}^{-\alpha_{\mathrm{S}} h}}{\alpha_{\mathrm{S}}} & \frac{-\gamma \mathrm{e}^{-\alpha_{\mathrm{S}} h}}{\alpha_{\mathrm{S}}}
\end{array}\right]
$$

where $\Delta_{\mathrm{P}}=\left(\Omega_{0}-\beta c\right)^{2} / c_{\mathrm{P}}^{2}$ and $\Delta_{\mathrm{S}}=\left(\Omega_{0}-\beta c\right)^{2} / c_{\mathrm{S}}^{2}$.

Stress matrix $[\mathrm{S}]$ 


$[S]=\mu\left[\begin{array}{llllll}\frac{2 i \alpha_{\mathrm{P}} \beta}{\Delta_{\mathrm{P}}} \mathrm{e}^{-\alpha_{\mathrm{P}} h} & -\frac{2 i \beta \alpha_{\mathrm{P}}}{\Delta_{\mathrm{P}}} & -\frac{\alpha_{\mathrm{S}}^{2}+\beta^{2}}{\alpha_{\mathrm{S}}} \mathrm{e}^{-\alpha_{\mathrm{P}} z} & \frac{-\beta \gamma}{\alpha_{\mathrm{S}}} & \frac{\alpha_{\mathrm{S}}^{2}+\beta^{2}}{\alpha_{\mathrm{S}}} & \frac{\beta \gamma}{\alpha_{\mathrm{S}}} \\ \frac{2 i \alpha_{\mathrm{P}} \gamma}{\Delta_{\mathrm{P}}} \mathrm{e}^{-\alpha_{\mathrm{P}} h} & -\frac{2 i \gamma \alpha_{\mathrm{P}}}{\Delta_{\mathrm{P}}} & \frac{-\beta \gamma}{\alpha_{\mathrm{S}}} & -\frac{\alpha_{\mathrm{S}}^{2}+\gamma^{2}}{\alpha_{\mathrm{S}}} \mathrm{e}^{-\alpha_{\mathrm{P}} h} & \frac{\beta \gamma}{\alpha_{\mathrm{S}}} & \frac{\alpha_{\mathrm{S}}^{2}+\gamma^{2}}{\alpha_{\mathrm{S}}} \\ \frac{i\left(2 \alpha_{\mathrm{S}}^{2}+\Delta_{\mathrm{S}}\right)}{\Delta_{\mathrm{P}}} \mathrm{e}^{-\alpha_{\mathrm{P}} h} & \frac{i\left(2 \alpha_{\mathrm{S}}^{2}+\Delta_{\mathrm{S}}\right)}{\Delta_{\mathrm{P}}} & -2 \beta \mathrm{e}^{-\alpha_{\mathrm{P}} z} & -2 \gamma \mathrm{e}^{-\alpha_{\mathrm{P}} h} & -2 \beta & -2 \gamma \\ -\frac{2 i \beta \alpha_{\mathrm{P}}}{\Delta_{\mathrm{P}}} & \frac{2 i \alpha_{\mathrm{P}} \gamma}{\Delta_{\mathrm{P}}} \mathrm{e}^{-\alpha_{\mathrm{P}} h} & \frac{\alpha_{\mathrm{S}}^{2}+\beta^{2}}{\alpha_{\mathrm{S}}} & \frac{\beta \gamma}{\alpha_{\mathrm{S}}} & -\frac{\alpha_{\mathrm{S}}^{2}+\gamma^{2}}{\alpha_{\mathrm{S}}} \mathrm{e}^{-\alpha_{\mathrm{P}} h} & -\frac{\beta \gamma}{\alpha_{\mathrm{S}}} \mathrm{e}^{-\alpha_{\mathrm{P}} h} \\ \frac{-2 i \gamma \alpha_{\mathrm{P}}}{\Delta_{\mathrm{P}}} & \frac{2 i \alpha_{\mathrm{P}} \gamma}{\Delta_{\mathrm{P}}} \mathrm{e}^{-\alpha_{\mathrm{P}} h} & \frac{\beta \gamma}{\alpha_{\mathrm{S}}} & \frac{\alpha_{\mathrm{S}}^{2}+\gamma^{2}}{\alpha_{\mathrm{S}}} & -\frac{\beta \gamma}{\alpha_{\mathrm{S}}} \mathrm{e}^{-\alpha_{\mathrm{P}} h} & -\frac{\alpha_{\mathrm{S}}^{2}+\gamma^{2}}{\alpha_{\mathrm{S}}} \mathrm{e}^{-\alpha_{\mathrm{P}} h} \\ -\frac{i\left(2 \alpha_{\mathrm{S}}^{2}+\Delta_{\mathrm{S}}\right)}{\Delta_{\mathrm{P}}} & -\frac{i\left(2 \alpha_{\mathrm{S}}^{2}+\Delta_{\mathrm{S}}\right)}{\Delta_{\mathrm{P}}} \mathrm{e}^{-\alpha_{\mathrm{P}} h} & 2 \beta & 2 \gamma & 2 \beta \mathrm{e}^{-\alpha_{\mathrm{P}} h} & 2 \gamma \mathrm{e}^{-\alpha_{\mathrm{P}} h}\end{array}\right]$

Stiffness matrix $[R]$ for half-space

$[R]=\frac{\mu}{\Delta}\left[\begin{array}{lll}\left(\beta^{2} \alpha_{\mathrm{P}}+\gamma^{2} \alpha_{\mathrm{S}}-\alpha_{\mathrm{P}} \alpha_{\mathrm{S}}^{2}\right) & \beta \gamma\left(\alpha_{\mathrm{S}}-\alpha_{\mathrm{P}}\right) & \beta\left(\alpha_{\mathrm{S}}^{2}+\beta^{2}+\gamma^{2}-2 \alpha_{\mathrm{P}} \alpha_{\mathrm{S}}\right) \\ \beta \gamma\left(\alpha_{\mathrm{S}}-\alpha_{\mathrm{P}}\right) & -2 \alpha_{\mathrm{P}} \gamma^{2}-\alpha_{\mathrm{P}} \beta^{2}+\alpha_{\mathrm{P}} \alpha_{\mathrm{S}}^{2} & \gamma\left(\alpha_{\mathrm{S}}^{2}+\beta^{2}+\gamma^{2}-2 \alpha_{\mathrm{P}} \alpha_{\mathrm{S}}\right) \\ \beta\left(\alpha_{\mathrm{S}}^{2}+\beta^{2}+\gamma^{2}-2 \alpha_{\mathrm{P}} \alpha_{\mathrm{S}}\right) & \gamma\left(\alpha_{\mathrm{S}}^{2}+\beta^{2}+\gamma^{2}-2 \alpha_{\mathrm{P}} \alpha_{\mathrm{S}}\right) & -\alpha_{\mathrm{S}}\left(\frac{\Omega_{0}-\beta c}{c_{\mathrm{S}}^{2}}\right)^{2}\end{array}\right]$

where $\Delta=\beta^{2}+\gamma^{2}-\alpha_{\mathrm{P}} \alpha_{\mathrm{S}}$.

[11] Krylov VV. Spectra of low frequency ground vibrations generated by high speed trains on layered ground. J Low Freq Noise Vib Active Control 1997;16(4):257-70.

[12] Gudehus G. A comprehensive constitutive equation for granular materials. Soils found 1996;36(1):1-12.

\section{References}

[1] Alabi B. A model for the problem of ground vibration induced by the wheels of a moving train. Appl Math Model 1989;13:710-5.

[2] Alabi B. A parametric study on some aspects of ground borne vibrations due to rail traffic. J Sound Vib 1992;153(1):77-87.

[3] De Barros FCP, Luco JE. Response of a layered visco-elastic half space to a moving point load. Wave Motion 1994;19:189-210.

[4] De Barros FCP, Luco JE. Stresses and displacements in a layered half space for a moving line load. Appl Math Comput 1995;67:103-34.

[5] Kausel E, Roesset JM. Stiffness matrices for layered soils. Bull Seismol Soc Am 1981;6:1743-61.

[6] Gunaratine M, Sanders O. Response of a layered elastic medium to a moving strip load. Int J Numer Anal Methods Geomech 1996;20: 191-208.

[7] Auersh L. Wave propagation in layered soils: theoretical solution in wave number domain and experimental results of hammer and railway traffic excitation. J Sound Vib 1994;173(2):233-64.

[8] Jones DV, Petyt M. Ground vibration in the vicinity of a rectangular load on a half space. J Sound Vib 1993;166(1):141-59.

[9] Jones DV, Petyt M. Ground vibration in the vicinity of a strip load: an elastic layer on an elastic half space. J Sound Vib 1993;161(1):1-18.

[10] Jones DV, Petyt M. Ground vibration in the vicinity of a strip load: an elastic layer on a rigid foundation. J Sound Vib 1992;152(3): 501-15.

[13] Lombaert G, Degrande G, François S, Kogut J, Pyl L. Validation of a numerical model for the prediction of railway induced vibrations in the free field. In: proceedings of the 11th international conference on earthquake geotechnical engineering, Berkeley, CA, USA, 7-9 January; 2004. p. 204-11.

[14] Hall L. Simulations and analyses of train-induced ground vibrations in finite element models. Soil Dyn Earthq Eng 2003;23:403-13.

[15] Andersen L, Nielsen SRK. Coupled finite element / boundary element analysis of a vehicle moving along a railway track. In: proceedings of the 11th international conference on earthquake geotechnical engineering, Berkeley, CA, USA, 7-9 January; 2004. p. 869-76.

[16] Massarsch KR. Mitigation of traffic-induced ground vibrations. In: proceedings of the 11th international conference on earthquake geotechnical engineering, Berkeley, C.A., USA, 7-9 January; 2004. p. 22-31.

[17] Sheng X, Jones CJC, Petyt M. Ground vibration generated by a load moving along a railway track. J Sound Vib 1999;228(1):129-56.

[18] Sheng X, Jones CJC, Thompson DJ. A comparison of a theoretical model for vibration from trains with measurements. In: proceedings of the seventh international workshop on railway noise. Portland, Maine, USA, 24-27 October; 2001, 8 pages.

[19] Grundmann H, Lieb M, Trommer E. The response of a layered half space to traffic load moving along its surface. Arch Appl Mech 1999; 69:55-67. 
[20] Jones D, Petyt M. Ground vibration in the vicinity of a strip load: a two dimensional half-space model. J Sound Vib 1991;147:155-66.

[21] Jones CJC, Sheng X, Petyt M. Simulation of ground vibration from a moving harmonic load on a railway track. J Sound Vib 2000;231(3): 739-51.

[22] Block JR, Temple BP. The influence of vehicle and track condition on the generation of railway ground borne vibration. In: seventh international congress on sound and vibration, July. Germany: Garmisch-Partenkirchen; 2000. p. 2719-726.

[23] Dinkel J, Trommer E, Grundmann H. Dynamics of vehicle tracksubgrade system - full track simulation model. In: conference on high speed trains on soft soils. Gothenburg, mars; 2000, 14 pages.

[24] Felszeghy SF. The Timoshenko beam on an elastic foundation and subjected to a moving step load. J Vib Acoust 1996;118:277-91.

[25] Paolucci R, Maffeis A, Scandella L, Stupazzini M, Vanini M. Numerical prediction of low-frequency ground vibrations induced by high-speed trains at Ledsgaard, Sweeden. Soil Dyn Earthq Eng 2003; 23:425-33.

[26] Dieterman HA, Metrikine AV. The equivalent stiffness of a half space interacting with a beam. Critical velocities of a moving load along the beam. Eur J Mech 1996;15(1):67-90.

[27] Krylov VV. Generation of ground vibrations by superfast trains. Appl Acoust 1995;44:149-64.

[28] Heckl MA, Hauck G, Wettschureck R. Structure borne sound and vibration from rail traffic. J Sound Vib 1996;193(1):175-84.

[29] Suiker ASJ, Chang C, De Borst R, Esveld C. Surface waves in a stratified half space with enhanced continuum properties. Part 1 . Formulation of the boundary value problem. Eur J Mech A Solids 1999;18:749-68.

[30] Suiker ASJ, Chang C, De Borst R, Esveld C. Surface waves in a stratified half space with enhanced continuum properties. Part 2. Analysis of the wave characteristics in regard to high speed railway tracks. Eur J Mech A Solids 1999;18:769-87.
[31] Lamb H. On the propagation of tremors over the surface of an elastic solid. Philos Trans Roy Soc London 1904;A203(1):1-42.

[32] Lefeuve-Mesgouez G. Propagation d'ondes dans un massif soumis à une charge se déplaçant à vitesse constante. $\mathrm{PhD}$ thesis (in French), Ecole Centrale of Nantes; 1999. 147 pages.

[33] Datoussaid S, De Saedeleer B, Verlinden O. Vehicle track interaction and ground propagation of vibrations for urban railway vehicles. Eur J Mech Environ Eng 1999;45(21):87-93.

[34] Picoux B. Le Houédec D. Propagation dans le sol des vibrations émises par un trafic ferroviaire se déplaçant à vitesse constante. Ministère de l'Aménagement du territoire et de l'Environnement. Aide $n^{\circ}$ DGAD/SRAE/99107. Final report (in French); 2001. 180 pages.

[35] Laghrouche O. Simulation numérique de propagations d'ondes dans les sols-Application à l'isolation vibratoire. $\mathrm{PhD}$ thesis (in French), Ecole Centrale of Nantes; 1996. 196 Pages.

[36] Lefeuve-Mesgouez G, Laghrouche O, Picoux B, Le Houedec D, Bettess P. Ground vibration in the vicinity of a moving harmonic strip load: semi analytical and numerical models. J Sound Vib, Submitted paper.

[37] Theodorakopoulos DD. Dynamic analysis of a poroelastic half-plane soil medium under moving loads. Soil Dyn Earthq Eng 2003;23: $521-33$.

[38] Theodorakopoulos DD, Chassiakos AP, Beskos DE. Dynamic effects of moving load on a poroelastic soil medium by an approximate method. Soil Dyn Earthq Eng 2004;41:1801-22.

[39] Schevenels M, Degrande G, Lombaert G. The influence of the depth of the ground water table on free field road traffic-induced vibrations. Int J Numer Anal Methods Geomech 2004;28:385-419.

[40] Picoux B. Etude théorique et expérimentale de la propagation des ondes dans le sol émise par un trafic ferroviaire. $\mathrm{Ph} \mathrm{D}$ thesis (in French), Ecole Centrale of Nantes, 002. 155 Pages. 